\title{
Super-Nernstian Tafel Slopes: An Origin in Coupled
}

\section{Homogeneous Kinetics}

\author{
Haotian Chen, Joseph R. Elliott*, Haonan Le, Minjun Yang and Richard G. Compton ${ }^{\dagger}$ \\ Department of Chemistry, Physical and Theoretical Chemistry Laboratory, Oxford University \\ South Parks Road, Oxford, OX1 3QZ, United Kingdom
}

\begin{abstract}
Simulations are reported relating to the predicted Tafel slopes of voltammograms recorded for heterogeneous electrochemically reversible electron transfer process coupled to non-linear homogeneous chemical kinetics. Tafel plots are shown to be markedly non-linear with potential indicating the essential need to be analysed via simulation. In the case of the $\mathrm{EC}_{2}$ reaction for a simple one-electron reversible electron transfer the following second order kinetics lead to Tafel slopes with apparent transfer coefficients notably above unity, so gain an apparent super-Nernstian response.Simulations for a 'titration' reaction mechanism, $A+e^{-} \rightleftharpoons B, B+C \rightarrow Y$ are also performed and the effects of electrode radius and analyte concentration on the Tafel slope are investigated and discussed.
\end{abstract}

Keywords: Voltammetry, Tafel equation, Simulation, Super-Nernstian responses, Non-linear homogeneous kinetics, $\mathrm{EC}_{2}$ and 'titration' reactions, Transfer coefficient

\section{Introduction}

A key component in the analysis of any current-voltage data embracing a Faradaic process is that of Tafel analysis, originating in the early 20th century work of Julius Tafel who, in the course of his work on electro-organic reactions, noted that plots of the logarithm of the Faradaic current, $I$, as a function of potential, $E$, were often linear at potentials near the onset of the reaction ${ }^{1 ; 2}$. Most recently IUPAC $^{3 ; 4}$ have defined, in the case of an electro-reduction, the cathodic Tafel transfer coefficient $\alpha$ to be:

\footnotetext{
${ }^{*}$ These authors contributed equally to this work.

†Corresponding author: Professor Richard G. Compton, richard.compton@chem.ox.ac.uk
} 


$$
\alpha=\frac{-\boldsymbol{R} \boldsymbol{T}}{F} \frac{d \ln (I)}{d E}
$$

with the corresponding definition for an anodic process, an electro-oxidation, as:

$$
\beta=\frac{\boldsymbol{R} \boldsymbol{T}}{F} \frac{d \ln (I)}{d E}
$$

where $\boldsymbol{R}$ is the gas constant, $\boldsymbol{T}$ is the temperature, and $F$ is the Faraday constant. Note that these definitions relate exclusively to the experimentally measured quantities $I$ and $E$. Earlier definitions involving the 'number of electrons transferred, n', and leading to expressions containing the term ' $n \alpha$ ', are eschewed, not least since $n$ may be unknown, difficult or impossible to measure, and in any case likely to be a function, for $n>1$, of the voltammetric timescale on which it is measured, so that different values of $n$ can be, and often are, seen at microelectrodes and at macroelectrodes.

In implementing equations (1) and (2) in respect of modern voltammetry - typically either cyclic voltammetry or steady-state voltammetry derived from a hydrodynamic or micro-electrode - it is necessary, in order that the Tafel slope or transfer coefficient reflects the heterogeneous electron transfer event, that the part of the voltammogram analysed is essentially free of mass transport limitations. The practical implication of this leads to the recommendation that no more than $30 \%$ of the maximum current of an electrochemical irreversible process be subject to Tafel analysis, a limit which has been recently recommended via numerical simulations ${ }^{5}$.

In the case of a simple one electron transfer involving two solution phase species,

$$
A+e^{-} \rightleftharpoons B
$$

the Tafel slope reflects the transfer coefficient within the Butler-Volmer formulation of electrode kinetics where, for an electron transfer which is slow compared with the prevailing mass transport,

$$
I \propto \exp \left(\frac{-\alpha F E}{\boldsymbol{R T}}\right)
$$

and $0<\alpha<1$; whilst for a multi-electron process,

$$
A+n e^{-} \rightleftharpoons B
$$

and if the reaction proceeds via a series of separate, non-concerted electron transfers and chemical steps, 


$$
I \propto \exp \left(-\frac{\left(n^{\prime}+\alpha_{R D S}\right) F E}{\boldsymbol{R T}}\right)
$$

where $n^{\prime}$ is the number of electrons transferred before the rate determining step and $\alpha_{R D S}$ is the transfer coefficient of the rate determining electron transfer step. In the later case that the measured Tafel slope indicates the value of $n^{\prime}+\alpha_{R D S}$. Both equations (3) and (4) predict an exactly linear Tafel plot of $\ln (I)$ versus $E$,in the absence of any depletion of the electroactive species.

In the Marcus-Hush theory of a simple one-electron transfer, in contrast, the transfer coefficient is not constant but is a function of applied potential giving rise to Tafel plots with a weak curvature, controlled by magnitude of the reorganization energy, $\lambda$, which has both inner and outer sphere contributions,

$$
\alpha=\frac{1}{2}+\frac{F\left(E-E_{f}^{0}\right)}{2 \lambda}
$$

where $E_{f, A / B}^{0}$ is the formal potential of the $A / B$ redox couple. Such curvature has been observed experimentally ${ }^{6-11}$. Equation 5 shows that $\alpha \approx 0.5$ at small overpotentials, which results from the Marcus-Hush theory in its simplest, symmetric form and assuming the curvature of the Gibbs energy parabola of the reactant $A$ and product $B$ to be the same ${ }^{12}$. In its asymmetric form the theory gives rise to values deviating from $\alpha \approx 0.5$ by an extent reflecting the difference in vibrational force constants in the $A$ and $B$ species ${ }^{13}$ as is, for example, thought to explain the low value of $\alpha$ seen for the reduction of di-oxygen to superoxide ${ }^{14}$.

In the case of fast (compared to prevailing mass transport), so-called reversible electrode kinetics, Tafel analysis shows for a simple, one-electron, reaction an "apparent" transfer coefficient of unity, where "apparent" cautions against relating this to Butler-Volmer or Marcus-Hush theories. In particular, Albery ${ }^{15}$ shows that for reversible steady-state voltammetry in which a limiting current $I_{l i m}$ is observed, for example at a rotating macro disc electrode or at a micro electrode, then for a reduction:

$$
\ln \left[\frac{1}{I}-\frac{1}{I_{\text {lim }}}\right]=+\frac{E F}{\boldsymbol{R T}}+\text { constant }
$$

In this situation the Tafel analysis with $\alpha=1$ simply shows the electron transfer is fast compared with mass transport. The process can be characterised as "Nernstian".

In this paper the Tafel analysis of electron-transfer reactions is further explored, notably in the electrochemically reversible limit, and, in particular, the effect of a homogeneous chemical reaction following an electron transfer is explored. First we confirm that there is a marked effect on the Tafel slope such that, as previously inferred ${ }^{16 ; 17}$, fast follow-up kinetics leads to irreversibility, since the rapid loss of 


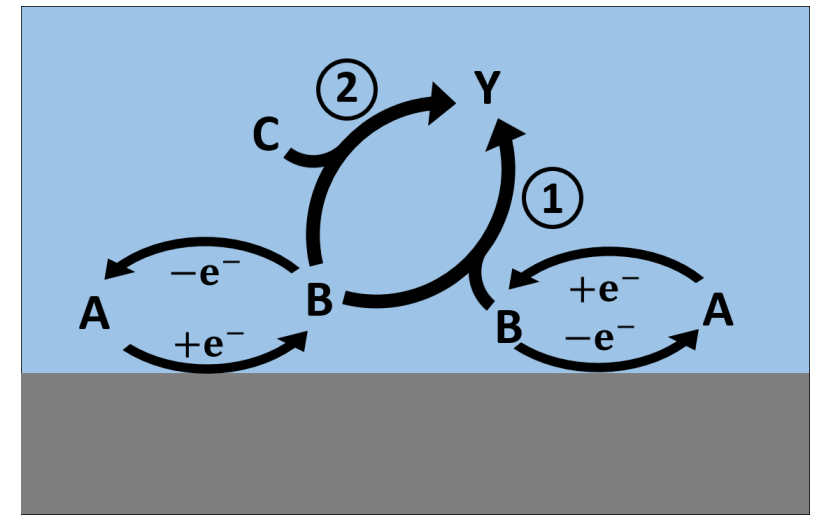

Figure 1: Illustration of the mechanisms investigated: (1) the $\mathrm{EC}_{2}$ mechanism, (2) the 'titration' mechanism. The electrode is represented by the grey area while the blue area shows the solution of phase.

B prevents Nernstian equilibrium being established, and hence the Tafel analysis reflects the electrontransfer kinetics of A alone. Second, and notably, we show that in the case of relatively slow second-order chemical reaction, the effect of the following chemical reaction is to increase the Tafel slope so that apparent transfer coefficients in excess of unity can be observed. This provides one explanation for the observation of "super-Nernstian" Tafel slopes even for the case of a rigorous one-electron process.

In order to identify "super-Nernstian" regimes, we especially focus on two cases (see Figure1). First the $\mathrm{EC}_{2}$ reaction,

$$
\begin{aligned}
& A+e^{-} \rightleftharpoons B \\
& B+B \stackrel{k_{2}}{\longrightarrow} Y
\end{aligned}
$$

where $k_{2}$ is the second order rate constant for $\mathrm{EC}_{2}$ reaction, and second a reaction in which electrogenerated $B$ reacts with a further species $C$, in bulk solution,

$$
\begin{aligned}
& A+e^{-} \rightleftharpoons B \\
& B+C \stackrel{k_{2}^{\prime}}{\longrightarrow} Y
\end{aligned}
$$

We refer to the latter as a 'titration' reaction, as $C$ is removed by reaction with $\mathrm{B}$ and is the basis of some types of electrochemical sensor, notably for phenols including tetrahydrocannabinol ${ }^{18}$, where the measurement of the oxidation/reduction of species Y provides a measure of the concentration of C. The next section develops the necessary theory for $\mathrm{EC}_{2}$ and titration reactions. 


\section{Theory}

This study primarily focuses on the electrochemically reversible, or quasi-reversible, heterogeneous, oneelectron reduction of species $A$ to $B$, followed by a homogeneous chemical step under diffusion-only mass transport. Planar and spherical electrode geometries are considered. The latter allows the transition between micro and macro-electrode to be readily explored.

The electrode kinetics are described using the Butler-Volmer theory of electrode kinetics, which states that, for a mechanism $A+e^{-} \rightleftharpoons B$ :

$$
j=k_{0} \exp \left[\frac{-\alpha F\left(E-E_{f}^{0}\right)}{\boldsymbol{R} \boldsymbol{T}}\right] c_{A}(0, t)-k_{0} \exp \left[\frac{(1-\alpha) F\left(E-E_{f}^{0}\right)}{\boldsymbol{R} \boldsymbol{T}}\right] c_{B}(0, t)
$$

where $j$ is the flux of the reductant on the electrode surface, $c_{A}(0, t)$ and $c_{B}(0, t)$ are the concentrations of species $A$ and $B$ at the electrode surface at time $t$ respectively, and $k_{0}$ is the standard electrochemical rate constant.

\section{$2.1 \quad \mathrm{EC}_{2}$ reaction model}

First, the $\mathrm{EC}_{2}$ reaction was simulated on a planar macroelectrode. By assuming that the scale of the electrode is significantly larger than the distance the species are able to diffuse over during the timescale of the simulation, the model is simplified to a one-dimensional system where only diffusion perpendicular to the surface of the electrode needs to be considered. In combination with Fick's Second Law of diffusion, the diffusion equation of this model can be expressed as:

$$
\frac{\partial c_{j}}{\partial t}=D_{j} \nabla^{2} c_{j}=D_{j} \frac{\partial^{2} c_{j}}{\partial x^{2}}
$$

where $j$ is the species under study and $D_{j}$ is the diffusion coefficient. For simplicity, during simulation, the diffusion coefficients of all species are set to be equal.

The rate of change in concentration with time in the solution phase can be expressed with Fick's Second Law of diffusion with appropriate terms accounting for the second-order homogeneous chemical reactions. See equation (7), when two $B$ molecules react and form $Y$, the process is irreversible and controlled by the second order rate constant, $k_{2}$ 


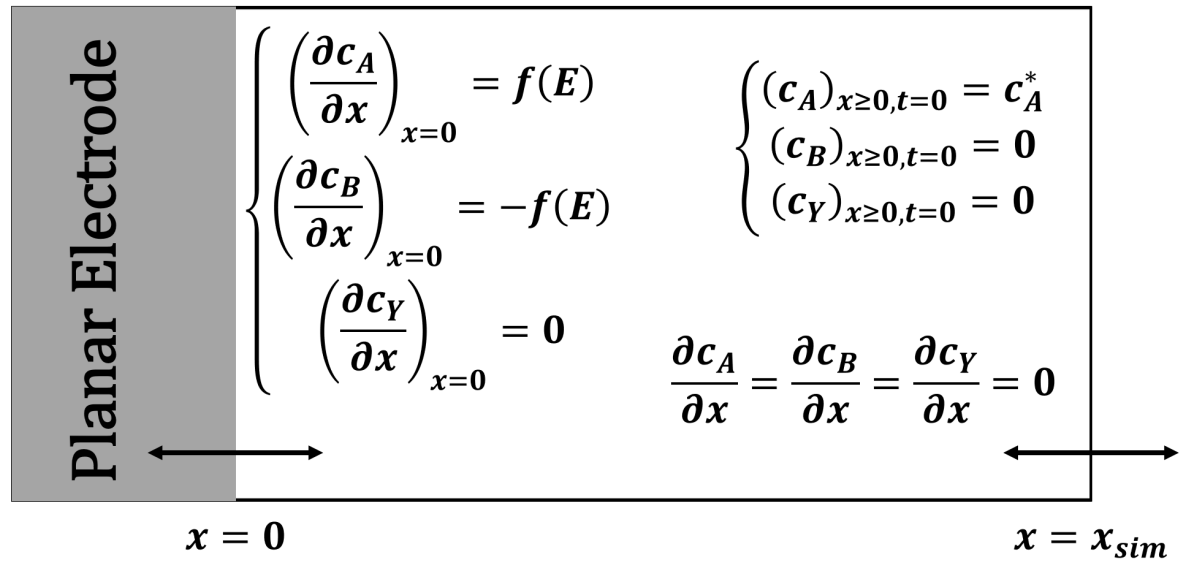

Figure 2: The boundary conditions used for the planar $\mathrm{EC}_{2}$ model. $c_{j}$ is the concentration of the species $j$ at a certain space point, $c_{A}^{*}$ is the initially homogeneous concentration of the analyte $A, x$ is the perpendicular distance from the electrode, $x_{\text {sim }}$ is the boundary of the simulated space in the x-axis and $f(E)$ is a function of the potential according to Butler-Volmer kinetics.

$$
\left\{\begin{aligned}
\frac{\partial c_{A}}{\partial t} & =D_{A} \frac{\partial^{2} c_{A}}{\partial x^{2}} \\
\frac{\partial c_{B}}{\partial t} & =D_{B} \frac{\partial^{2} c_{B}}{\partial x^{2}}-k_{2} c_{B}^{2} \\
\frac{\partial c_{Y}}{\partial t} & =D_{Y} \frac{\partial^{2} c_{Y}}{\partial x^{2}}+\frac{1}{2} k_{2} c_{B}^{2}
\end{aligned}\right.
$$

To solve these differential equations, boundary conditions at the start of the simulation and at both spatial boundaries (the electrode surface and outer boundary) must be defined (see also Figure 2). The initial concentration of $A$ in bulk solution is set to $c_{A}^{*}$ with no $B$ or $Y$ present in the system.

$$
t=0, x \geq 0\left\{\begin{array}{l}
c_{A}=c_{A}^{*} \\
c_{B}=0 \quad \text { and } \quad c_{A}^{*} \geq 0 \\
c_{Y}=0
\end{array}\right.
$$

where $x$ approaches $x_{\text {sim }}$, on outer spatial boundary, the concentration of each species equals its initial bulk concentration. This boundary needs to be sufficiently far from the electrode that changes in concentration at its surface have no effect in the simulation on the concentration at the upper boundary. The size of the Nernst diffusion layer, $\delta$, depends on time as $\delta \propto D t$. It is understood from Einstein's work on Brownian motion ${ }^{20}$ that in one dimension for a particle: $\sqrt{\left\langle x^{2}\right\rangle}=\sqrt{2 D t_{\text {sim }}}$, which can be used to approximate the distance from the electrode at which one might expect concentration perturbations to persist. The distance of the outer boundary from the electrode, $x_{\text {sim }}$, in the simulations, is selected to 
be greater than this $^{21}$ :

$$
x_{\text {sim }}=6 \sqrt{D t_{\text {sim }}}
$$

The boundary conditions at the surface of electrode reflect the electroactivity of the species in the potential range of study:

$$
\left\{\begin{array}{l}
D_{A}\left(\frac{\partial c_{A}(x, t)}{\partial x}\right)_{x=0}=k_{0} e^{-\alpha \frac{F\left(E-E_{f}^{0}\right)}{R T}} c_{A}(0, t)-k_{0} e^{(1-\alpha) \frac{F\left(E-E_{f}^{0}\right)}{R T}} c_{B}(0, t) \\
D_{B}\left(\frac{\partial c_{B}(x, t)}{\partial x}\right)_{x=0}=-D_{A}\left(\frac{\partial c_{A}(x, t)}{\partial x}\right)_{x=0} \\
\left(\frac{\partial c_{Y}(x, t)}{\partial x}\right)_{x=0}=0
\end{array}\right.
$$

where $A$ and $B$ are electro-active and follow Butler-Volmer kinetics, while $Y$ is electro-inactive and has zero flux at the electrode.

\section{2 'Titration' reaction model}

The 'titration' reaction was simulated by considering a spherical electrode. This was done to allow both macro- and micro-electrode limits to be explored. For spherical electrodes, spherical coordinates $(r, \theta$, $\phi)$ are used. As the system is symmetric with respect to both angles $\theta$ and $\phi$, Fick's Second Law in the spherical geometry can be reduced to one dimension for species $\mathrm{j}$ :

$$
\frac{\partial c_{j}}{\partial t}=D_{j}\left(\frac{\partial^{2} c_{j}}{\partial r^{2}}+\frac{2}{r} \frac{\partial c_{j}}{\partial r}\right)
$$

The heterogeneous reduction of $A$ to $B$ at the electrode surface is followed by a homogeneous chemical reaction in solution between the electrogenerated B and an electro-inactive 'titrate' species already present in solution, $C$. The resulting chemical product, $Y$, is considered electro-inactive. See equation (8), the reaction is irreversible and controlled by the rate constant $k_{2}^{\prime}$. The diffusion equations, with the homogeneous chemical reaction considered, are shown below:

$$
\left\{\begin{array}{l}
\frac{\partial c_{A}}{\partial t}=D_{A}\left(\frac{\partial^{2} c_{A}}{\partial r^{2}}+\frac{2}{r} \frac{\partial c_{A}}{\partial r}\right) \\
\frac{\partial c_{B}}{\partial t}=D_{B}\left(\frac{\partial^{2} c_{B}}{\partial r^{2}}+\frac{2}{r} \frac{\partial c_{B}}{\partial r}\right)-k_{2}^{\prime} c_{B} c_{C} \\
\frac{\partial c_{C}}{\partial t}=D_{C}\left(\frac{\partial^{2} c_{C}}{\partial r^{2}}+\frac{2}{r} \frac{\partial c_{C}}{\partial r}\right)-k_{2}^{\prime} c_{B} c_{C} \\
\frac{\partial c_{Y}}{\partial t}=D_{Y}\left(\frac{\partial^{2} c_{Y}}{\partial r^{2}}+\frac{2}{r} \frac{\partial c_{Y}}{\partial r}\right)+k_{2}^{\prime} c_{B} c_{C} \\
D_{A}=D_{B}=D_{C}=D_{Y}
\end{array}\right.
$$




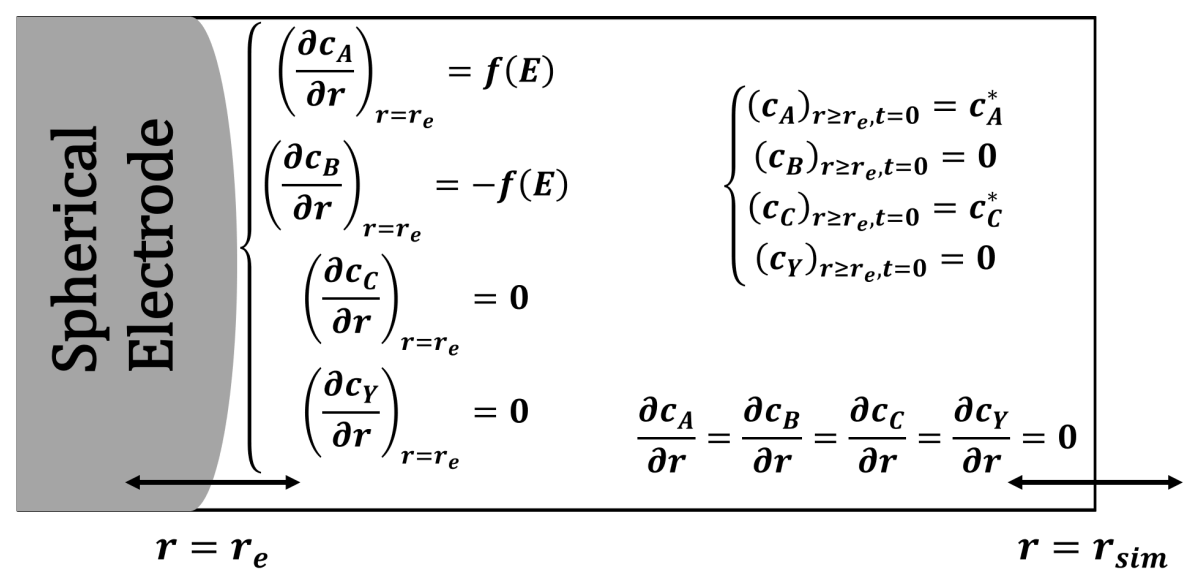

Figure 3: Illustration of the boundary conditions used for the spherical 'titration' model. $c_{j}$ is the concentration of the species $j$ at a certain space, $c_{A}^{*}$ is the initially homogeneous concentration of the analyte $A, c_{C}^{*}$ is the initially homogeneous concentration of the species $C, r$ is the radial distance from the centre of the electrode, $r_{e}$ is the radius of the electrode, $r_{s i m}$ is the radial boundary of the simulated space and $f(E)$ is a function of the potential according to Butler-Volmer kinetics. Each of the boundary conditions is explored in greater detail in the body of the text.

The simulation boundary conditions are defined as shown in Figure 3. Note that $r=0$ denotes the centre of the interior of the electrode and $r=r_{e}$ denotes the electrode surface, which is the start of the simulation space. The bulk concentrations of $A$ and $C$ are set to be arbitrary values at the beginning of the simulation. The temporal and outer boundary conditions are thus:

$$
t=0, r \geq r_{e}\left\{\begin{array}{l}
c_{A}=c_{A}^{*} \\
c_{B}=0 \\
c_{C}=c_{C}^{*} \\
c_{Y}=0
\end{array} \quad \text { and } \quad c_{A}^{*} \geq 0, c_{C}^{*} \geq 0\right.
$$

Similarly to the $\mathrm{EC}_{2}$ model, the outer boundary of simulation is defined at $r_{\text {sim }}=r_{e}+\sqrt{6 D t_{\text {sim }}}$. The boundary conditions at the electrode surface are described using the Butler-Volmer theory of electrode kinetics for the electroactive $A$ and $B$, while a zero-flux condition is imposed for the electro-inactive $C$ and $Y$ : 


$$
\left\{\begin{array}{l}
D_{A}\left(\frac{\partial c_{A}(r, t)}{\partial r}\right)_{r=r_{e}}=k_{0} e^{-\alpha \frac{F\left(E-E_{f}^{0}\right)}{R T}} c_{A}\left(r_{e}, t\right)-k_{0} e^{(1-\alpha) \frac{F\left(E-E_{f}^{0}\right)}{R T}} c_{B}\left(r_{e}, t\right) \\
D_{B}\left(\frac{\partial c_{B}(r, t)}{\partial r}\right)_{r=r_{e}}=-D_{A}\left(\frac{\partial c_{A}(r, t)}{\partial r}\right)_{r=r_{e}} \\
\left(\frac{\partial c_{C}(r, t)}{\partial r}\right)_{r=r_{e}}=0 \\
\left(\frac{\partial c_{Y}(r, t)}{\partial r}\right)_{r=r_{e}}=0
\end{array}\right.
$$

\subsection{Dimensionless parameters}

For simplicity and generality of simulation, dimensionless parameters are adopted and listed in Table 1.

Rewriting the continuity equations and boundary conditions for the $\mathrm{EC}_{2}$ model into dimensionless coordinates gives:

$$
\begin{gathered}
\left\{\begin{array}{l}
\frac{\partial C_{A}}{\partial T}=d_{A} \frac{\partial^{2} C_{A}}{\partial X^{2}} \\
\frac{\partial C_{B}}{\partial T}=d_{B} \frac{\partial^{2} C_{B}}{\partial X^{2}}-K_{2} C_{B}^{2} \\
\frac{\partial C_{Y}}{\partial T}=d_{Y} \frac{\partial^{2} C_{Y}}{\partial X^{2}}+\frac{1}{2} K_{2} C_{B}^{2}
\end{array}\right. \\
T=0, X \geq 0 \\
T>0, X \rightarrow X_{s i m}\left\{\begin{array}{c}
C_{A}=1 \\
C_{B}=0 \\
C_{Y}=0
\end{array}\right. \\
\left\{\begin{array}{l}
\left(\frac{\partial C_{A}(X, T)}{\partial X}\right)_{X=0}=K_{0} e^{-\alpha \theta} C_{A}(0, T)-K_{0} e^{(1-\alpha) \theta} C_{B}(0, T) \\
d_{B}\left(\frac{\partial C_{B}(X, T)}{\partial X}\right)_{X=0}=-\left(\frac{\partial C_{A}(X, T)}{\partial X}\right)_{X=0} \\
\left(\frac{\partial C_{Y}(X, T)}{\partial X}\right)_{X=0}=0
\end{array} \quad \begin{array}{l}
\text { and } \quad J=\left(\frac{\partial C_{A}(X, T)}{\partial X}\right)_{X=0} \\
\end{array}\right.
\end{gathered}
$$

Similarly, rewriting for the titration reaction model gives:

$$
\left\{\begin{array}{l}
\frac{\partial C_{A}}{\partial T}=\left(\frac{\partial^{2} C_{A}}{\partial R^{2}}+\frac{2}{R} \frac{\partial C_{A}}{\partial R}\right) \\
\frac{\partial C_{B}}{\partial T}=d_{B}\left(\frac{\partial^{2} C_{B}}{\partial R^{2}}+\frac{2}{R} \frac{\partial C_{B}}{\partial R}\right)-K_{2}^{\prime} C_{B} C_{C} \\
\frac{\partial C_{C}}{\partial T}=d_{C}\left(\frac{\partial^{2} C_{C}}{\partial R^{2}}+\frac{2}{R} \frac{\partial C_{C}}{\partial R}\right)-K_{2}^{\prime} C_{B} C_{C} \\
\frac{\partial C_{Y}}{\partial T}=d_{Y}\left(\frac{\partial^{2} C_{Y}}{\partial R^{2}}+\frac{2}{R} \frac{\partial C_{Y}}{\partial R}\right)+K_{2}^{\prime} C_{B} C_{C}
\end{array}\right.
$$




$$
T>0, R \geq 1\left\{\begin{array}{l}
C_{A}=1 \\
C_{B}=0 \\
C_{C}=C_{C}^{*} \\
C_{Y}=0
\end{array} \quad \text { and } \quad C_{C}^{*}=1\right.
$$

$$
\begin{cases}\left(\frac{\partial C_{A}(R, T)}{\partial R}\right)_{R=1}=K_{0} e^{-\alpha \theta} C_{A}(1, T)-K_{0} e^{(1-\alpha) \theta} C_{B}(1, T) & \\ d_{B}\left(\frac{\partial C_{B}(R, T)}{\partial R}\right)_{R=1}=-\left(\frac{\partial C_{A}(R, T)}{\partial R}\right)_{R=1} & \text { and } \quad J=\left(\frac{\partial C_{A}(R, T)}{\partial R}\right)_{R=1} \\ \left(\frac{\partial C_{C}(R, T)}{\partial R}\right)_{R=1}=0 & \\ \left(\frac{\partial C_{Y}(R, T)}{\partial R}\right)_{R=1}=0 & \end{cases}
$$

The calculation of the apparent transfer coefficient is expressed as:

$$
\alpha_{\text {apparent }}=-\frac{d \ln \left|J_{\text {red }}\right|}{d \theta}
$$


Table 1: List of definitions of dimensionless parameters for planar and spherical electrode simulations. $c_{j}$ is the concentration of species $j$ and $c_{A}^{*}$ is the bulk concentration of species $A$. $\epsilon$ is the radius of the planar electrode, assumed to be the flat surface of a circular disc, area $\pi \epsilon^{2}$, and $r_{e}$ is the radius of the spherical electrode.

\begin{tabular}{|c|c|c|}
\hline Dimensionless Parameter & Definition (Planar) & Definition (Spherical) \\
\hline Concentration of species $j$ & $C_{j}=\frac{c_{j}}{c_{A}^{*}}$ & $C_{j}=\frac{c_{j}}{c_{A}^{*}}$ \\
\hline Diffusion coefficient of species $\mathrm{j}$ & $d_{j}=\frac{D_{j}}{D_{A}}$ & $d_{j}=\frac{D_{j}}{D_{A}}$ \\
\hline Spatial Coordinates & $X_{i}=\frac{x_{i}}{\epsilon}$ & $R_{i}=\frac{r_{i}}{r_{e}}$ \\
\hline Time & $T=\frac{D_{A} t}{\epsilon^{2}}$ & $T=\frac{D_{A} t}{r_{e}^{2}}$ \\
\hline Potential & $\theta=\frac{F}{R T}\left(E-E_{f}^{0}\right)$ & $\theta=\frac{F}{R T}\left(E-E_{f}^{0}\right)$ \\
\hline Scan Rate & $\sigma=\frac{\epsilon^{2}}{D_{A}} \frac{F}{R T} \nu$ & $\sigma=\frac{r_{e}^{2}}{D_{A}} \frac{F}{R T} \nu$ \\
\hline Current & $J=\frac{I}{\pi \epsilon F D_{A} C_{A}^{*}}$ & $J=\frac{I}{4 \pi r_{e} F D_{A} C_{A}^{*}}$ \\
\hline Rate Constant at Formal Potential (Butler-Volmer) & $K_{0}=\frac{k_{0} \epsilon}{D_{A}}$ & $K_{0}=\frac{k_{0} r_{e}}{D_{A}}$ \\
\hline Chemical Rate Constant, Second Order & $K_{2}=\frac{k_{2} c_{A} * \epsilon^{2}}{D_{A}}$ & $K_{2}^{\prime}=\frac{k_{2}^{\prime} c_{A}^{*} r_{e}^{2}}{D_{A}}$ \\
\hline
\end{tabular}

\subsection{Simulation methods}

The simulations were performed using home written $C++$ programs with OpenMP and std::thread for parallel computing. The diffusion equation was solved using the finite difference method by discretising the dimensionless diffusion equation into an expanding space grid $^{22}$ and using the backward implicit method $^{23}$. To solve the multi-diagonal sparse matrix given by the implicit method, the root-finding Newton-Raphson method was applied. Note that the Newton-Raphson method was iterated until the average error was smaller than $10^{-10}$ for the $\mathrm{EC}_{2}$ model or smaller than $10^{-8}$ for the titration model. The resulting voltammograms were verified by ensuring the error of mass conservation was below $10^{-10 \%}$ and thorough testing ${ }^{24-26}$ was performed using convergence testing, and comparison with theoretical peak currents and peak heights. The plotting was carried out on OriginLab 2017, Matlab 2019b and in Python with the packages NumPy, SciPy and matplotlib. 


\section{Results and discussion}

In this section, the simulated results with the corresponding analysis and discussion for the the two models are presented. The simulated cyclic voltammograms of $\mathrm{EC}_{2}$ reaction model are shown first to generate "super-Nernstian" Tafel slopes.

\section{$3.1 \mathrm{EC}_{2}$ reaction model}

Two sets of simulations were performed for the $\mathrm{EC}_{2}$ reaction model for slow (irreversible) and fast (reversible) electrode kinetics of the electrochemical reaction by setting $K_{0}$ to $10^{-3}$ and $10^{8}$, respectively, at a fixed dimensionless scan rate $\sigma=38943$, which corresponds to the dimensional scan rate $\nu=1 \mathrm{~V} / \mathrm{s}$ for the dimensional parameters of $\epsilon=1 \mathrm{~mm}, c_{A}^{*}=1 \mathrm{mM}, D_{A}=10^{-5} \mathrm{~cm}^{2} \mathrm{~s}^{-1}$ and $\alpha=0.5$. Irreversible and reversible Randles-Ševčík equations ${ }^{27 ; 28}$ were used to validate the simulation model and compare with the peak currents of the simulated voltammograms:

$$
\left\{\begin{array}{l}
\left|I_{p I}\right|=0.496 \sqrt{\alpha} F A c_{A}^{*} \sqrt{\frac{F D \nu}{\boldsymbol{R T}}} \\
\left|I_{p R}\right|=0.446 F A c_{A}^{*} \sqrt{\frac{F D \nu}{\boldsymbol{R T}}}
\end{array}\right.
$$

where $I_{p I}$ is the predicted peak current for irreversible Randles-Ševčík equation, $I_{p R}$ is the predicted peak current for the reversible Randles-Ševčík equation, and $A$ is the electrode area. By varying the chemical reaction kinetics $K_{2}$ for both slow and fast electrode kinetics, a marked dependence of the simulated cyclic voltammograms on $K_{2}$ is seen and presented in Figure 4. 

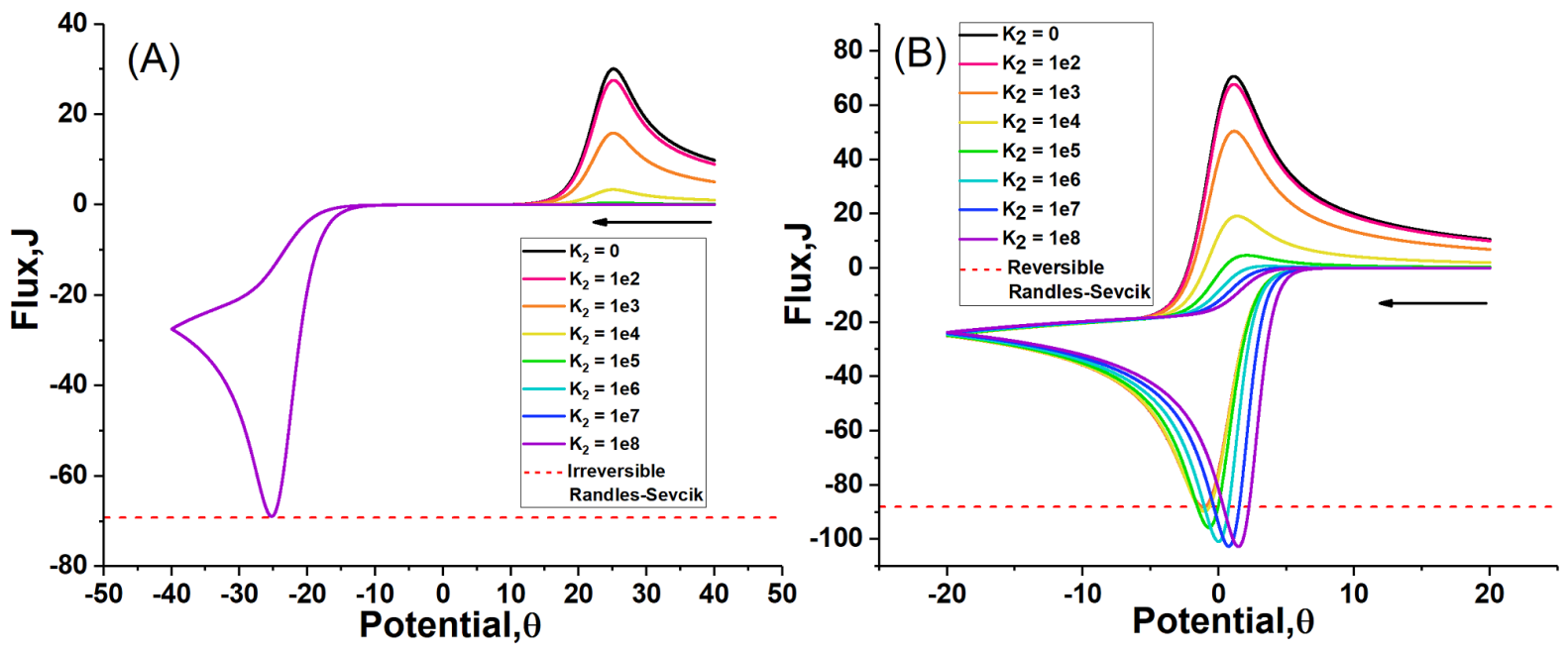

Figure 4: Cyclic Voltammograms of the slow $(A)$ and fast $(B)$ electrochemical kinetics $K_{0}$ for different values of chemical kinetics $K_{2}$ in the $\mathrm{EC}_{2}$ reaction model. The red dashed lines in figures represent the values of peak flux predicted by the irreversible (A) and reversible (B) Randles-Ševčík equations. The solid lines represent the cyclic voltammograms for different $K_{2}$. The black arrows indicate the start and initial direction of the potential sweep.

As shown in Figure $4 A$, the forward peak is almost unaffected as the $K_{2}$ value increases, so the apparent transfer coefficient stays at a similar level. See Figure $5 A, \alpha \approx 0.5$, for slow regimes of electrode kinetics $K_{0}$ since the electron transfer is 'blind' to the following kinetics. However, the back peak of the voltammogram decreases when $K_{2}$ increases for the slow electrode kinetics reflecting the loss of B via homogeneous chemistry.

In Figure $4 B$, for the reversible case, by increasing $K_{2}$, not only does the back peak of the voltammogram decreases, the forward peak flux increases as the forward peak position moves towards more positive potentials. See Figure $5 B$, the value of $\alpha$ also changes: in the range of $K_{2}$ simulated in Figure $4 \mathrm{~B}$,the forward scan peak becomes "sharper" as $K_{2}$ increases, and $\alpha$ increases from unity at $K_{2}=0$ to a maximum of 1.4 at $K_{2}=10^{8}$.

The decrease in the back peak fluxes for both the fast electrode kinetics and the slow reflects the fact that larger $K_{2}$ increases the chemical irreversibility of the electrochemical reaction as the increasing $K_{2}$ results in a decreased amount of species $B$ in the system after the forward scan. Whilst, the forward scan of the electrochemical reaction of an irreversible process is independent of the further reaction of species $B$, in contrast, the changes in forward peak shape in Figure $4 B$ as $K_{2}$ increases can be explained by the fact that, as the $K_{2}$ increases, the concentration of $B$ is reduced by the homogeneous chemical reaction, 

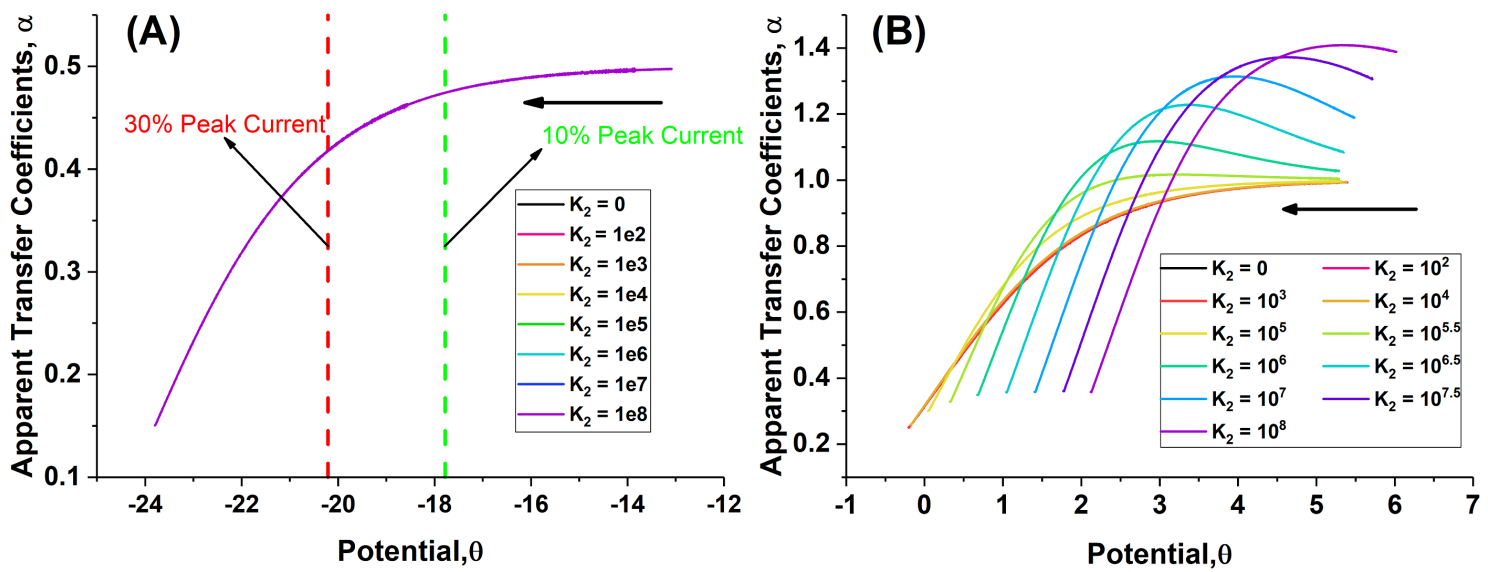

Figure 5: Figure A shows the apparent transfer coefficients as a function of potential extracted from $\mathrm{EC}_{2}$ reactions with slow electrode kinetics at $K_{0}=10^{-3}$ as shown in Figure $4 \mathrm{~A}$. $0.1 \%$ to $99 \%$ of peak current is analysed and plotted. The positions of $10 \%$ to $30 \%$ peak current are plotted with vertical lines on this figure. Figure $\mathrm{B}$ is the apparent transfer coefficients extracted from $\mathrm{EC}_{2}$ reactions with fast electrode kinetics at $K_{0}=10^{8}$ as shown in Figure 4B with the follow up chemical reaction rate constants from 0 to $10^{8}$. Note that more values of $K_{2}$ than shown in Figure $4 \mathrm{~B}$ are considered for better visualization. The range of the apparent transfer coefficients to be analysed is from $0.1 \%$ to $99 \%$ of the peak current. The black arrows indicate the start and initial direction of the potential sweep.

which drives the reaction from $A$ towards $B$ and leads to a less negative starting potential of the forward peak and larger magnitude of the peak flux.

To further investigate the changes in the apparent transfer coefficients $\alpha$ of the volatmmograms for slow $\left(K_{0}=10^{-3}\right)$ and fast electrode kinetics $\left(K_{0}=10^{8}\right)$, the values of $\alpha$ are extracted as shown in Figure $5 \mathrm{~A}$ and $5 \mathrm{~B}$, by calculating the derivative of natural $\log$ of flux, $\ln J$ over potential, $\theta$, using:

$$
\alpha=\lim _{\Delta \theta \rightarrow 0} \frac{\ln J_{(\theta+\Delta \theta)}-\ln J_{\theta}}{\Delta \theta}
$$

In Figure 5A, the apparent transfer coefficients for the irreversible process stays at around 0.5 at less reductive potentials and decreases to around 0.15 near the peak of the forward scan due to change of concentration at the electrode surface and mass transport. The apparent transfer coefficient curves for different $K_{2}$ overlap with each other, indicating that the follow up homogeneous chemical reaction does not significantly affect the behaviour of forward scan peaks, and thus leaves the transfer coefficients unaffected. But this behaviour is very different for the reversible process, as discussed below.

In Figure 5B, the apparent transfer coefficients increases once $K_{2}$ reaches around $10^{5}$ and "superNernstian" behaviour appears when $K_{2}>10^{5.5}$. The increasing apparent transfer coefficients are consistent with the a "sharper" forward scan peak at higher $K_{2}$ as shown in Figure 4B. The curves in Figure 
5B show two patterns. At low $K_{2}$, when the rate of follow up chemical reaction is slow compared with electrode kinetics, apparent transfer coefficients curves are not significantly affected, and the curve behaves like a simple one-electron reduction, as the apparent transfer coefficients curve steadily decreases from around unity at the onset of reduction to lower values at 0.3. However, when $K_{2}$ increases, the apparent transfer coefficients increases first at higher potential, and then drops as the potential shifts to more reductive values. As explained above, the fast removal of reduced B from A by homogeneous chemical reaction requires less reductive potential for the start of reduction and thus the super-Nernstian curves are at higher potential when $K_{2}$ is large.

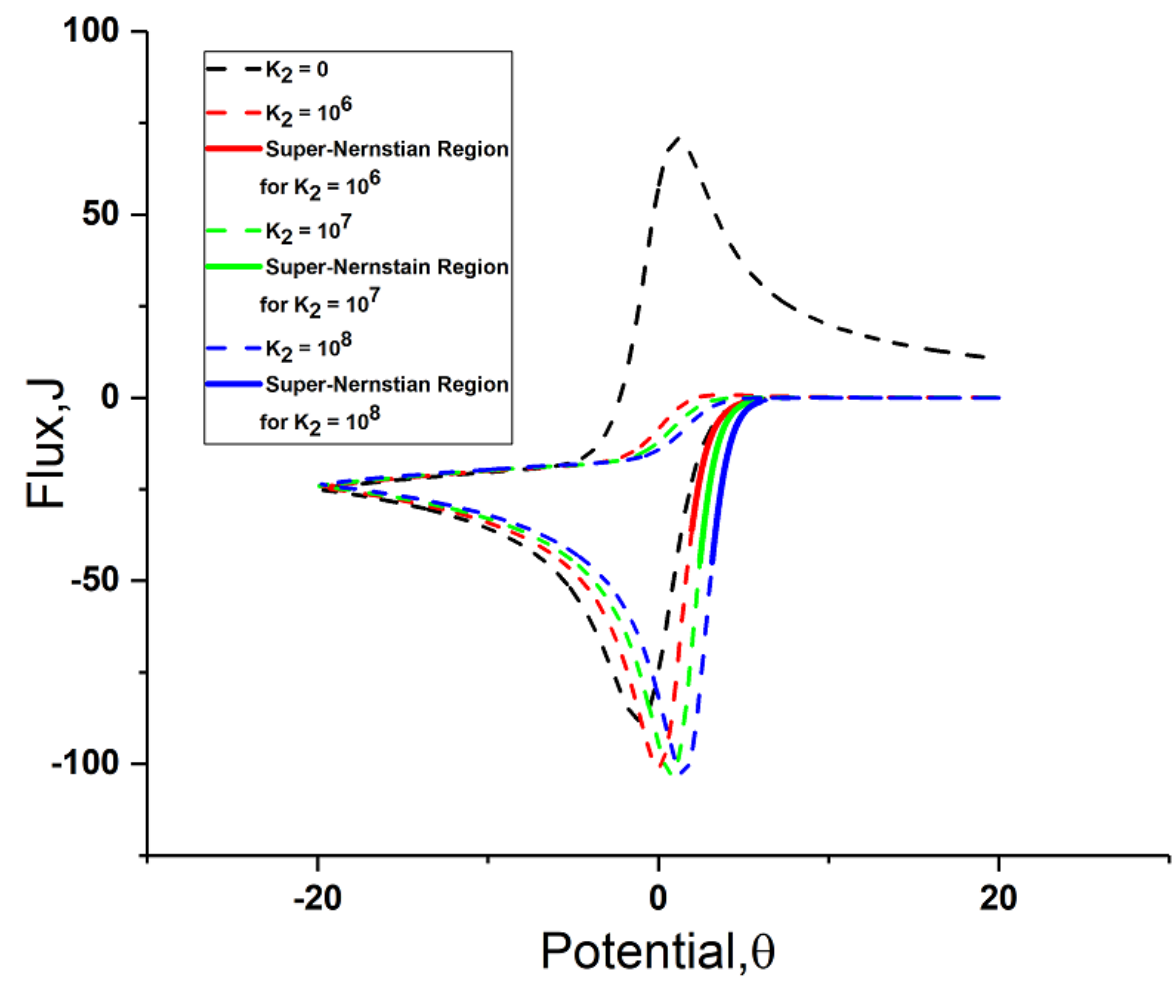

Figure 6: Among the four voltammograms(dash lines) selected from Figure 4B, the region of voltammogram where exists super-Nernstian behaviours is highlighted with solid lines.

To better appreciate the super-Nernstian behavior in a voltammogram, the portion of the voltammogram with an apparent transfer coefficients greater than unity is labeled with a solid line as shown in Figure 6. Four cyclic voltammograms are shown: a reference of $K_{2}=0$ and three voltammograms with fast chemical kinetics of $K_{2}=10^{6}, 10^{7}, 10^{8}$. It can be seen that a super-Nernstian responses can be seen at potentials typically used for Tafel analysis and that they are maximal for intermediate values of $K_{2}$ between very fast and relatively slow. Note that $K_{2}$ depends on $C^{*}$ that the studied experimental ranges 
of $K_{2}$ can be adjusted accordingly.

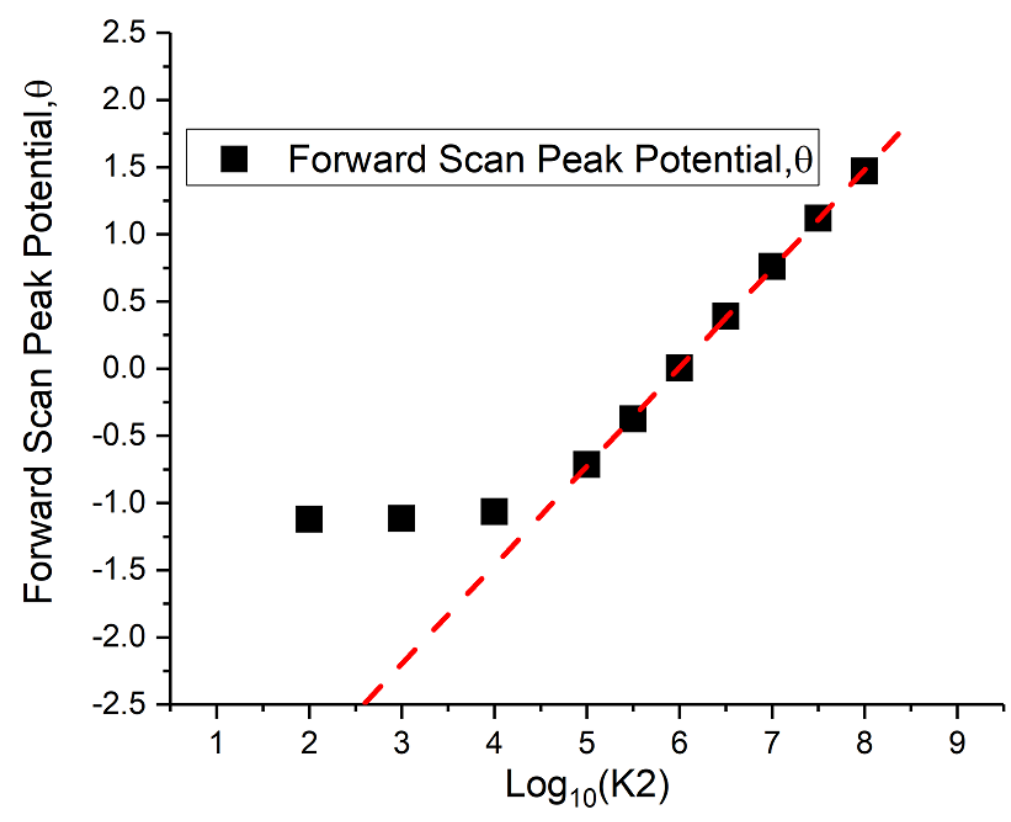

Figure 7: At $K_{0}=10^{8}, \sigma=38943$, the forward scan peak potential at different $K_{2}$ is plotted. When $K_{2}$ is greater than $10^{5}$, there is a strong linear relationship between $\log _{10} K_{2}$ and $\theta$. When $K_{2}=0$, the peak potential is constant at -1.12 .

The cathodic peak potential can also provide insights into the voltammograms as shown in Figure 7 for the fully reversible limit. When $K_{2}$ is smaller than $10^{5}$, the peak potential stays around -1.12 . But when $K_{2}$ reaches around $10^{5}$, the forward scan potential starts to increase. Beyond this point exists a linear relationship between $\log _{10} K_{2}$ and forward scan peak potential, $\theta_{\text {peak }}$. The linear relationship can be expressed as $\theta_{\text {peak }}=0.736 \log _{10}\left(K_{2}\right)-4.402$ and the $R^{2}$ value is 0.999 , indicating a strong linear correlation between $\log _{10} K_{2}$ and peak potential $\theta_{\text {peak }}$. With this equation, the forward scan peak position at high $K_{2}$ can be easily inferred.

The increase in value of $\alpha$ from $K_{2}=10^{5}$ can be attributed to the same fact that the reduction in the concentration of $B$ drives the electrode reaction to generate more $B$. The second-order following kinetics means that the loss is increasingly promoted at more negative potentials as the kinetic term becomes relatively more important relative to transport.

Note that simulations were also carried out for a simple EC process $\left(A+e^{-} \leftrightharpoons B, B \rightarrow C\right)$, that is with first order following kinetics. No such super-Nernstian behaviours were seen. Rather as the following rate constant increases, when the electrode kinetics are fast, the apparent Tafel slope changes monotonically from unity to $\alpha=\frac{1}{2}$, the value set in the simulation. This reflects the linearity of the system so that changing concentration influences both diffusion and chemical reaction proportionally. 
Dimensional mapping The apparent transfer coefficient is dependent on various parameters including some of the key dimensional parameters: scan rate, $k_{0}$, and $k_{2}$. In order to establish a simulation model that can be used to predict the value of $\alpha$ under the various influences of these parameters in a $\mathrm{EC}_{2}$ system, the values of apparent $\alpha$ are extracted for voltammograms of various $\sigma$ and $K_{2}$ for three different electrode kinetics $\left(K_{0}=10^{4}, 10^{2}, 10^{0}\right.$, which correspond to $\left.k_{0}=10^{0}, 10^{-2}, 10^{-4} \mathrm{~cm} / \mathrm{s}\right)$ at $(\epsilon=1 \mathrm{~mm}$, $c_{A}^{*}=1 \mathrm{mM}, D_{A}=10^{-5} \mathrm{~cm}^{2} \mathrm{~s}^{-1}$ and $\left.\alpha=0.5\right)$ shown in Figure 8. The range of $K_{2}$ in these simulations is from $K_{2}=1.0$ to $k_{0}=10^{10}$, and corresponds to $k_{2}=1.0 M^{-1} s^{-1}$ to $k_{2}=10^{10} M^{-1} s^{-1}$. The range of dimensionless scan rate $\sigma$ is from $\sigma=3.8943$ to $\sigma=3.8943 \times 10^{6}$, and corresponds to practical experimental scan rate of $10^{-4} \mathrm{~V} / \mathrm{s}$ to $10^{2} \mathrm{~V} / \mathrm{s}$ with the other dimensional parameters mentioned above. The apparent transfer coefficients are calculated based on a more common experimental approach by finding linear fitting the natural logarithm of $10 \%$ to $30 \%$ of peak flux with potential and the fitted slope is taken as the apparent transfer coefficients ${ }^{5}$. For better illustration, the plots are presented in dimensional parameters as the scan rate and $k_{2}$ are chosen relate to experiments at practice.

As presented in Figure 8, the value of $\alpha$ decreases as the scan rate increases for all three $k_{0}$ if $k_{2}$ is kept constant. The value of $\alpha$ increases as $k_{2}$ increases before it reaches a maximum roughly 1.3 for smaller scan rates (up to $10^{-2} \mathrm{~V} / \mathrm{s}$ in $A$ and $10^{-3} \mathrm{~V} / \mathrm{s}$ in $B$ ). However, for larger scan rates (from $10^{-2} \mathrm{~V} / \mathrm{s}$ to $10^{2} \mathrm{~V} / \mathrm{s}$ in $A$, from $10^{-3} \mathrm{~V} / \mathrm{s}$ to $10^{2} \mathrm{~V} / \mathrm{s}$ in $B$, and all scan rates in $B$ ), the value increases as $k_{2}$ increases before reaching a maximum and subsequently decreasing. Therefore, a comprehensive set of $\alpha$ maps is provided to help understand and predict the value of $\alpha$ in an $\mathrm{EC}_{2}$ system.

Further guidance for experimentalists $\mathrm{In}$ this $\mathrm{EC}_{2}$ model, the dimensionless homogeneous chemical kinetics $K_{2}$ is dependent not only on its intrinsic dimensional rate constant $k_{2}$, but also on the concentration of species $A$. Therefore, altering $c_{A}^{*}$ can assist experimentalists to observe a "super-Nernstian" $\alpha$ for a system with a known $k_{2}$. As shown in Figure 9, the values of $\alpha$ are shown for a selection of $k_{2}\left(10^{1}\right.$, $\left.10^{3}, 10^{6}, 10^{9} \mathrm{M}^{-1} \mathrm{~s}^{-1}\right)$ for three different $k_{0}\left(10^{0}, 10^{-2}, 10^{-4} \mathrm{~cm} / \mathrm{s}\right)$ at different scan rates.

For all the curves with $c_{A}^{*}=1 m M$ in Figure 9 (solid lines), as $k_{2}$ increases, the $\alpha$ values for all scan rates and $k_{0}$ increase until a maximum occurs $\left(k_{2}=10^{1} M^{-1} s^{-1}\right.$ for $A$ and $B, k_{2}=10^{1} M^{-1} s^{-1}$ for $C)$. Then the $\alpha$ decreases except for simulations at extremely high scan rates $\left(k_{2}=10^{9} \mathrm{M}^{-1} \mathrm{~s}^{-1}\right.$ for $A$ and $B$ ). Additionally, the $\alpha$ values for higher scan rates decreases as $k_{0}$ decreases. As for simulations with $c_{A}^{*}=10 \mathrm{mM}$ in Figure 9 (dashed lines), for those with larger $k_{2}$ than the maximum discussed above $\left(k_{2}=10^{1} M^{-1} s^{-1}\right.$ for $A$ and $B, k_{2}=10^{1} M^{-1} s^{-1}$ for $C$ ), their values of $\alpha$ decrease compared to their counterparts at $c_{A}^{*}=1 \mathrm{mM}$ except high scan rates of $k_{0}=10^{-4} \mathrm{~cm} / \mathrm{s}$. Otherwise, the values of $\alpha$ for the other situations increases as concentration increase except for high scan rates for all three $k_{0}$. Overall, 

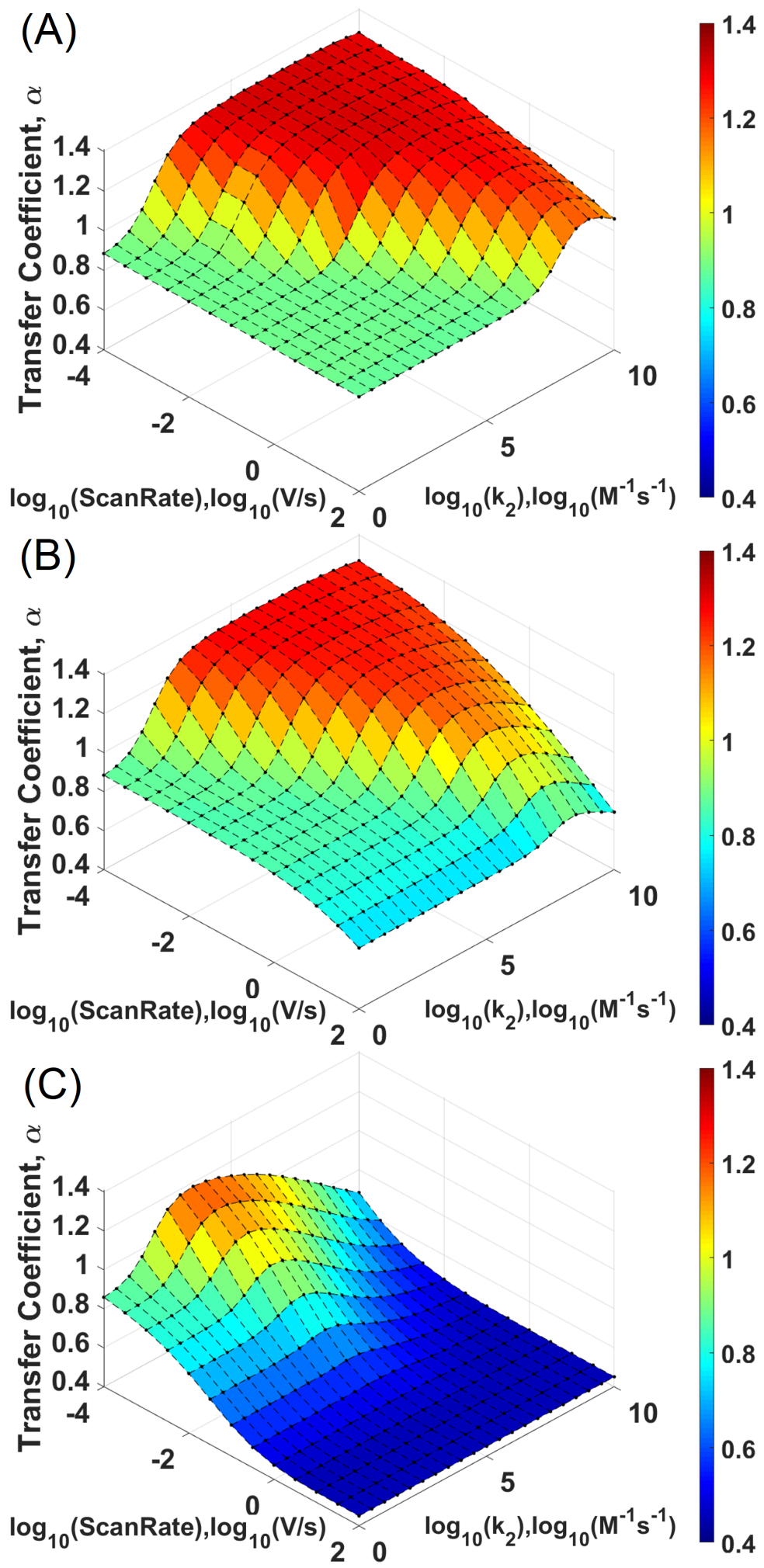

Figure 8: Apparent transfer coefficients $\alpha$ obtained from cyclic voltammograms for various scan rate $\sigma$ and the homogeneous chemical kinetics $k_{2}$ at $k_{0}=10^{0}(A), 10^{-2}(B), 10^{-4} \mathrm{~cm} \mathrm{~s}^{-1}(C)$ with corresponding dimensional parameter at $\left(\epsilon=1 \mathrm{~mm}, c_{A}^{*}=1 \mathrm{mM}, D_{A}=10^{-5} \mathrm{~cm}^{2} \mathrm{~s}^{-1}\right.$ and $\left.\alpha=0.5\right)$. The homogeneous chemical kinetics dimension, $k_{2}$, is from 1 to $10^{10} M^{-1} s^{-1}$, corresponding to dimensionless $K_{2}$ from 1 to $10^{10}$. The dimensional scan rate is from $10^{-4}$ to $10^{2} \mathrm{~V} / \mathrm{s}$, corresponding to dimensionless scan rate $\sigma$ from 3.8943 to $3.8943 \times 10^{6}$. 

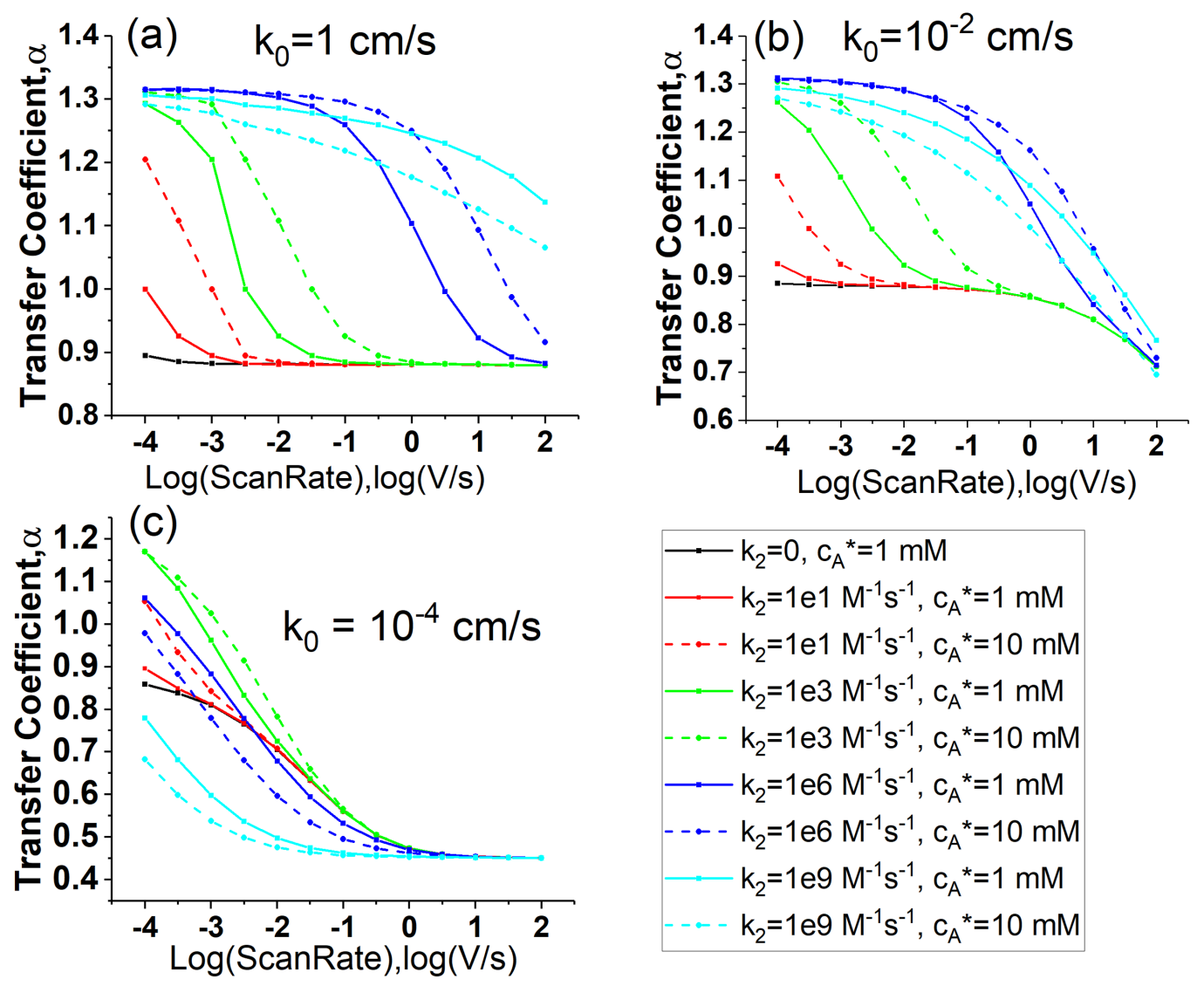

Figure 9: Apparent transfer coefficients $\alpha$ obtained from cyclic voltammograms for various scan rate $\sigma$ and the homogeneous chemical kinetics $k_{2}$ for $c_{A}^{*}=1 \mathrm{mM}$ (solid lines), $10 \mathrm{~m} M$ (dashed lines) at $k_{0}=10^{0}(A)$, $10^{-2}(B), 10^{-4} \mathrm{~cm} / \mathrm{s}(C)$.

in practice, one can observe a higher value of $\alpha$, even "super-Nernstian" $\alpha$, by simply adjusting the concentration of the starting material $A$.

\section{2 'Titration' Reaction Model}

In this section we examine the 'titration' reaction to again explore the influence of following chemical kinetics on the measured Tafel slope for the A/B voltammetric wave. First, the effect of varying $K_{2}^{\prime}$ for the reversible and irreversible limits, with $C_{C}^{*}=1$ and under planar diffusion conditions, is explored. Second, the effect of varying $C_{C}^{*}=c_{C}^{*} / c_{A}^{*}$ under the same conditions is investigated.

In order to compare with the data in the preceding section, we first simulate cyclic voltammograms with a relatively fast dimensionless scan rate under which the diffusion of the analyte is considered to be planar. This allows direct comparison with the planar $\mathrm{EC}_{2}$ model and also the Randles-Ševčík equations. Figure 10 presents two sets of voltammograms with electrode kinetics set as 'irreversible' $\left(K_{0}=10^{-2}\right)$ 
or 'reversible' $\left(K_{0}=10^{5}\right)$. The dimensionless scan rate is chosen to be 38943 , which corresponds to $1 \mathrm{~V} / \mathrm{s}$ for a spherical electrode of radius $1 \mathrm{~mm}, \alpha=0.5$, and diffusion coefficients of $D=10^{-5} \mathrm{~cm}^{2} / \mathrm{s}$. The dimensionless $K_{2}^{\prime}$ range between $10^{2}$ and $10^{8}$ corresponds to a $k_{2}^{\prime}$ range from $10^{2}$ to $10^{8} M^{-1} s^{-1}$ under the same conditions. The initial concentration of species $\mathrm{C}$ is set equal to the inital concentration of species A, therefore $C_{C}^{*}=1$.
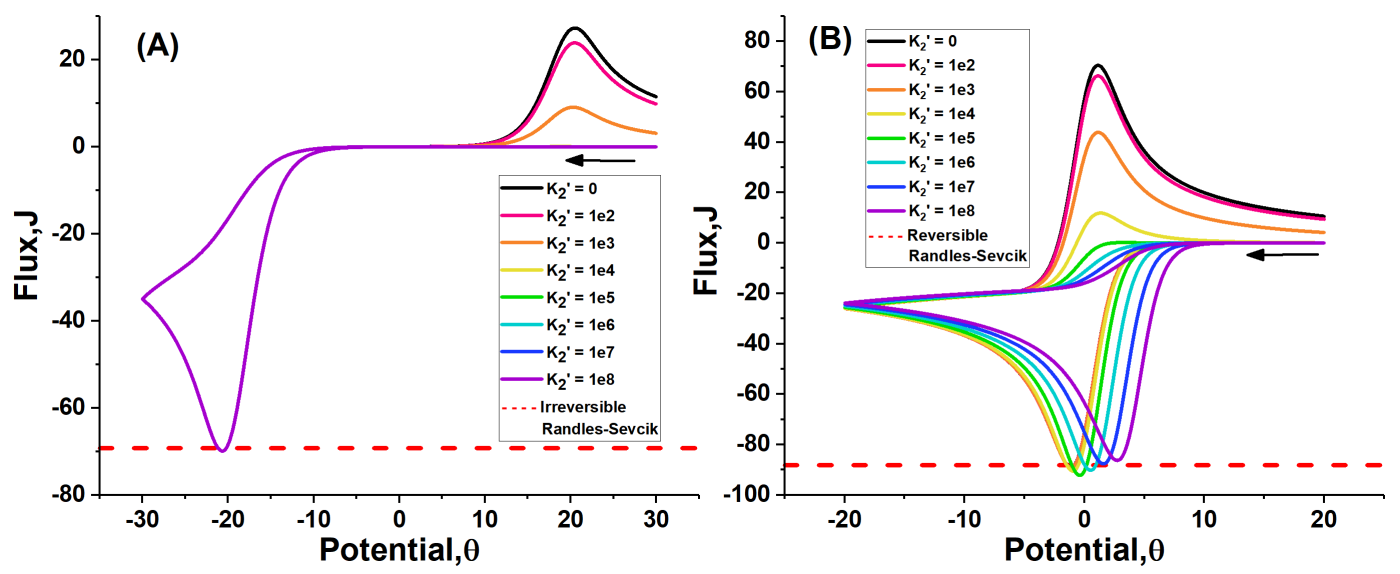

Figure 10: Cyclic voltammograms of the slow $(A)$ and fast $(B)$ electrochemical kinetics $K_{0}$ for different values of chemical kinetics $K_{2}^{\prime}$ in the titration reaction model with $C_{C}^{*}=1$. The red dashed lines in figures represent the values of peak flux predicted by the irreversible(A) and reversible(B) RandlesŠevčík equations. The solid lines represent the cyclic voltammograms for different $K_{2}^{\prime}$. The black arrows indicate the start and initial direction of the potential sweep.

Qualitatively similar features are seen as in Figure 4. Thus the following kinetics, varied over the range $K_{2}^{\prime}=0$ to $10^{8}$, have negligible impact on the forward peak current in the irreversible limit where equation (26) accurately predicts the simulated value. Further, in both cases, as expected, the back peak is increasingly diminished as $K_{2}^{\prime}$ gets larger. In the reversible limit the dimensionless forward peak potential, $\theta_{f p}$, is shifted to less negative potentials, as seen with the case of the simple $\mathrm{EC}_{2}$ reaction. Figure 11 shows a plot of $\theta_{f p}$ vs. $\log _{10} K_{2}^{\prime}$. The dimensionless peak potential stays near -1.12 when $K_{2}^{\prime}$ is smaller than $10^{4}$ but begins to increase for greater $K_{2}^{\prime}$. A linear relationship develops between $\log _{10}\left(K_{2}^{\prime}\right)$ and the forward scan peak potential, $\theta_{f p}$ for $K_{2}^{\prime}>10^{6}$ and can be expressed as $\theta_{f p}=1.139 \log _{10}\left(K_{2}^{\prime}\right)-6.376$, with a value of the coefficient of determination, $R^{2}$, of 0.99998 .

The impact of the following kinetics is weakened in the case of the titration reaction as compared to the $\mathrm{EC}_{2}$ process as is evident in the apparent transfer coefficients $\alpha_{\text {apparent }}$ calculated from $\frac{d \ln |J|}{d \theta}$, shown in Figure 12 for fast electrode kinetics $\left(K_{0}=10^{5}\right)$ and $C_{C}^{*}=1$ as a function of $\theta$.

In Figure 12(A), as was the case for the $\mathrm{EC}_{2}$ mechanism presented in Figure 5(A), the apparent transfer coefficients for the irreversible process remain unchanged by an increasing $K_{2}^{\prime}$, indicating that 


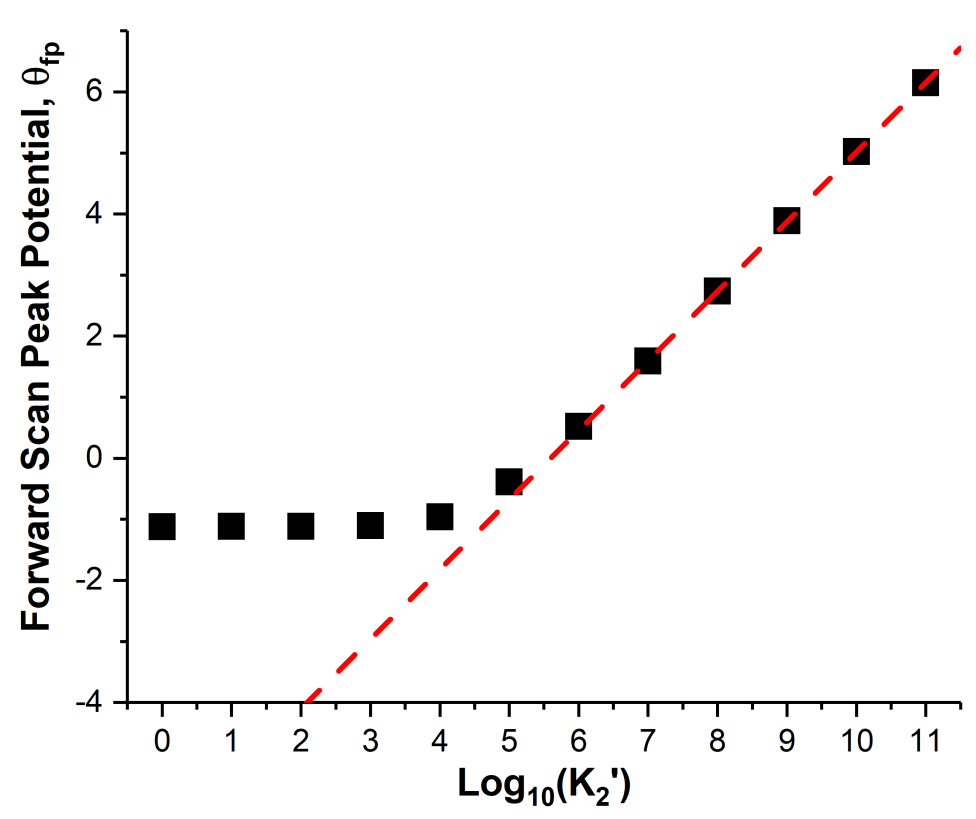

Figure 11: At $K_{0}=10^{5}, \sigma=38943$, and $C_{C}^{*}=1$, the forward scan peak potential at different $K_{2}^{\prime}$ is plotted. When $K_{2}^{\prime}$ is greater than $10^{6}$, there is a linear relationship between $\log _{10} K_{2}^{\prime}$ and $\theta$, as shown with the red dotted line.
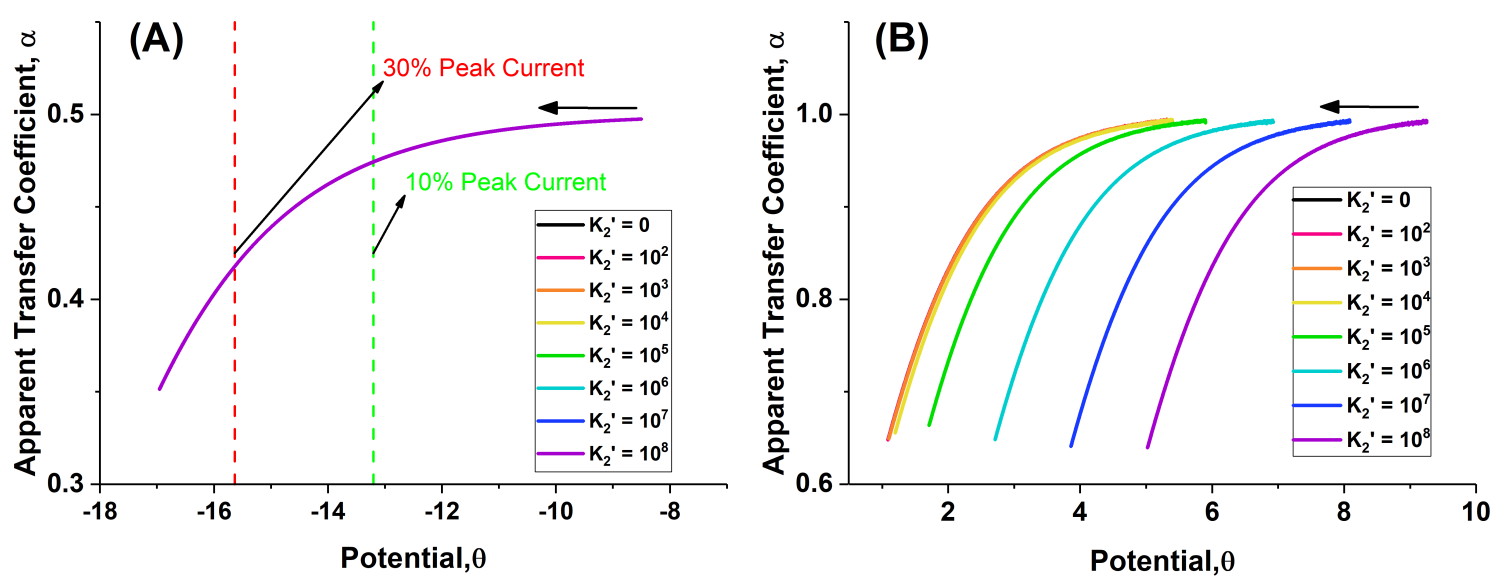

Figure 12: Figure (A) shows the apparent transfer coefficient as a function of potential extracted from titration reactions with slow electrode kinetics at $K_{0}=10^{-2}, \sigma=38943$, and $C_{C}^{*}=1$. $1 \%$ to $50 \%$ of peak current is analysed and plotted. The potentials corresponding to $10 \%$ to $30 \%$ peak current are plotted with vertical lines on this figure. Figure (B) shows the apparent transfer coefficient extracted from titration reactions with fast electrode kinetics at $K_{0}=10^{5}, \sigma=38943$, and $C_{C}^{*}=1$ with the follow up chemical reaction rate constants from 0 to $10^{8}$. The range of the apparent transfer coefficients to be analysed is from $1 \%$ to $50 \%$ of the peak current. Savitzky-Golay smoothing ${ }^{29 ; 30}$ was performed for both Figures to reduce artificial noise at large under-potentials. The black arrows indicate the direction of the potential sweep.

the follow up homogeneous chemical reaction does not, as expected, significantly affect the behaviour of the forward scan peaks. 
However, the behaviour is again different for the reversible case, as shown in Figure 12(B). The apparent transfer coefficients do not change significantly with $K_{2}^{\prime}$ until $K_{2}^{\prime} \approx 10^{4}$; the following kinetics are not sufficiently fast to disturb the electrochemical system. For $K_{2}^{\prime} \geqslant 10^{4}$, increasing $K_{2}^{\prime}$ causes the potentials analysed to become significantly more positive as the peak shifts due to the depletion of B shifting the equilibrium. The apparent transfer coefficient for each $K_{2}^{\prime}$ varies with potential, $\theta$, from $\alpha_{\text {apparent }} \approx 0.99$ at $1 \%$ of the peak current to $\alpha_{\text {apparent }} \approx 0.65$ at $50 \%$ of the peak current.

In particular, there is no significant change in the shape of these transfer coefficient curves (unlike in Figure 5 for the $\mathrm{EC}_{2}$ mechanism): the curves for the titration mechanism remain monotonic and do not enter any super-Nernstian regime for $C_{C}^{*}=1$. Nevertheless, their curvatures do vary slightly: the transfer coefficient curves' curvature decreases with increasing $K_{2}^{\prime}$ from around $K_{2}^{\prime}=10^{3}$ to around $10^{5}$, after which it increases with increasing $K_{2}^{\prime}$. This can be evidenced by the variation in the value of $\alpha_{\text {apparent }}$ at $50 \%$ of the peak current with $K_{2}^{\prime}$, despite having similar $\alpha_{\text {apparent }}$ values at $1 \%$ of the peak current and similar $\theta$-ranges.

The effect of varying $C_{C}^{*}$ In this section we investigate the effects of changing the bulk concentration of $\mathrm{C}$ relative to the bulk concentration of $\mathrm{A}, C_{C}^{*}$, for the case of fast electrochemical kinetics, namely increasing $C_{C}^{*}$ to 10 and then decreasing $C_{C}^{*}$ to 0.1 . To allow direct comparison, each of the other dimensionless parameters is kept identical: $K_{0}=10^{5}, \sigma=38943$ and $K_{2}^{\prime}=0$ to $10^{11}$. This $K_{2}^{\prime}$ range corresponds to dimensional values of $k_{2}^{\prime}$ from 0 to $10^{11} M^{-1} s^{-1}$ for a spherical electrode with a radius of $1 \mathrm{~mm}$ and diffusion coefficients of $D=10^{-5} \mathrm{~cm}^{2} / \mathrm{s}$, encapsulating the full range of physical values.
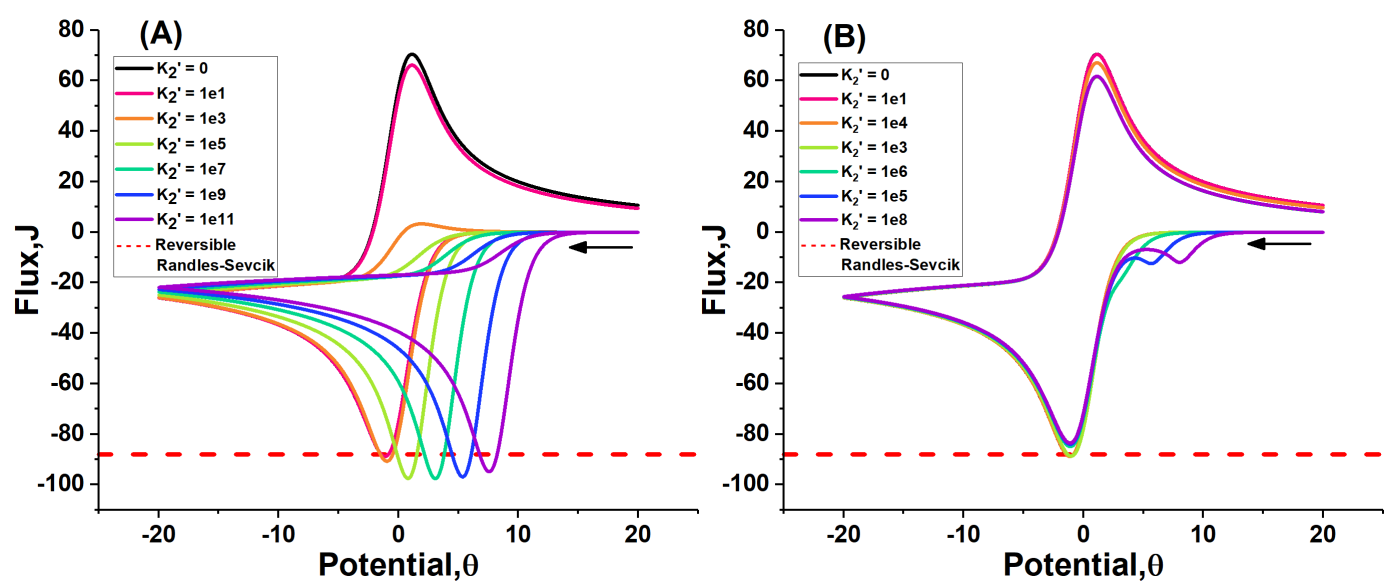

Figure 13: Cyclic Voltammograms of $C_{C}^{*}=10(\mathrm{~A})$ and $C_{C}^{*}=0.1(\mathrm{~B})$ under fast electrochemical kinetics $K_{0}$ for different values of chemical kinetics $K_{2}^{\prime}$ in the titration reaction model. The red dashed lines represent the values of peak flux predicted by the reversible Randles-Ševčík equations. The solid lines represent the cyclic voltammograms for different $K_{2}^{\prime}$. The black arrows indicate the start and initial direction of the potential sweep. 

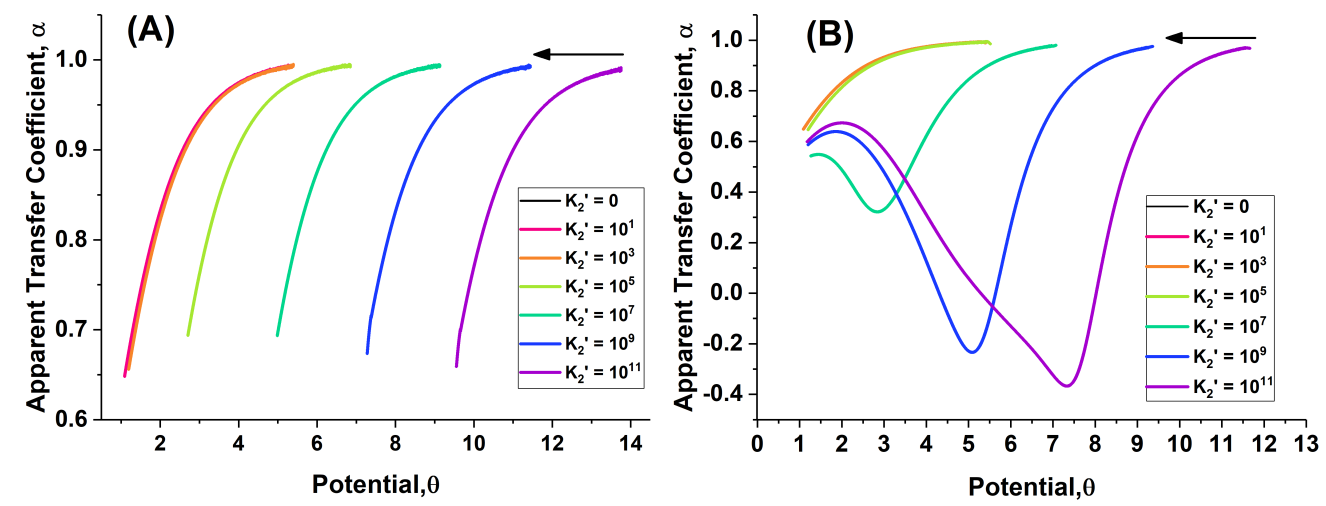

Figure 14: Figure (A) shows the apparent transfer coefficient as a function of potential extracted from titration reactions with fast electrode kinetics and increased $C_{C}^{*}: K_{0}=10^{5}, \sigma=38943$, and $C_{C}^{*}=10$ with following chemical reaction rate constants, $K_{2}^{\prime}$, from 0 to $10^{11}$. $1 \%$ to $50 \%$ of the peak current is analysed and plotted. Figure (B) shows the apparent transfer coefficient extracted from titration reactions with fast electrode kinetics and decreased $C_{C}^{*}: K_{0}=10^{5}, \sigma=38943$, and $C_{C}^{*}=0.1$ with following chemical reaction rate constants, $K_{2}^{\prime}$ from 0 to $10^{11}$. The range of the apparent transfer coefficients to be analysed is from $1 \%$ to $50 \%$ of the peak current. For $K_{2}^{\prime}$ values where a pre-peak is present in the cyclic voltammogram, i.e. $K_{2}^{\prime}=10^{9}$ and $10^{11}$, the base-line was set at the minimum in $|J|$ produced by the pre-peak. SavitzkyGolay smoothing ${ }^{29 ; 30}$ was performed for both Figures to reduce noise at highly positive potentials. The black arrows indicate the direction of the potential sweep.

Increasing $C_{C}^{*}$ from 1 to 10 (whilst keeping all other parameters identical) has an interesting effect for the case of fast electrochemical kinetics on how the following kinetics affect the voltammogram (Figure $13(\mathrm{~A})$ ). We see that the range of $K_{2}^{\prime}$ in which the peak current is increased from the value predicted by the reversible Randles-Ševčík equation lasts to higher $K_{2}^{\prime}$ than for $C_{C}^{*}=1$. The increase in $C_{C}^{*}$ also leads to a greater variation between the transfer coefficient curves for different $K_{2}^{\prime}$, as seen in Figure 14(A). The average value of $\alpha_{\text {apparent }}$ in the range of $1-50 \%$ peak current increases from the value for $K_{2}^{\prime}=0$ and remains larger throughout the $K_{2}^{\prime}$ range.

Figure 13(B) demonstrates how decreasing $C_{C}^{*}$ from 1 to 0.1 causes a pre-peak to form and the main peak current to drop for fast $K_{2}^{\prime}$. The pre-peak forms when the following kinetics shift the reduction of A to less negative potentials but its size is limited by the small, finite amount of $\mathrm{C}$ present such that the latter is fully 'titrated' by reaction with electrogenerated B. The depletion of $C_{C}$ in the vicinity of the electrode outpaces the mass transport during the timescale of the forward sweep as $C_{C}^{*}=0.1$, leading to the formation of a pre-peak. The early influence of the chemical kinetics reduces the amount of A present at the electrode, such that, once the potential is negative enough to reduce A without the influence of the following chemical kinetics, the main peak current is reduced.

Decreasing $C_{C}^{*}$ below 1 has a considerable effect on the plot of $\alpha_{\text {apparent }}$ against $\theta$ (Figure 14(B)) as calculated from equation 25. For large values of $K_{2}^{\prime}$, capable of greatly disturbing the electrochemical 
system, notably $K_{2}^{\prime} \geq 10^{7}$, the depletion of $\mathrm{C}$ produces a minimum in $\alpha_{\text {apparent }}$. As $K_{2}^{\prime}$ increases, this $\alpha_{\text {apparent }}$ minimum decreases in value and shifts to more positive $\theta$, such that, once a distinct pre-peak is formed, $\alpha_{\text {apparent }}$ can become negative (since $\frac{d|J|}{d \theta}>0$ immediately after the pre-peak), as observed for $K_{2}^{\prime}=10^{9}$ and $10^{11}$.

Looking at low potentials, we see a maximum in the $\alpha_{\text {apparent }}$. As $K_{2}^{\prime}$ increases, the depletion of $\mathrm{C}$ occurs at more positive potentials, thus increasing the separation of the pre- and main peaks. As a result, we see that the $\alpha_{\text {apparent }}$ maximum increases in value and shifts to more positive potentials.

Micro-electrode regime Last we examine the effect of the transition from planar to convergent/divergent diffusion by simulating the 'titration' reaction at a micro-sphere electrode. We consider the case of a $1 \mu m$ radius spherical electrode, with $\sigma=0.038943, K_{0}=10^{2}$, and $K_{2}^{\prime}=0$ and $10^{-5}$ to $10^{5}$, for $C_{C}^{*}$ values of 10, 1, and 0.1, as shown in Figure 15. These dimensionless parameters are chosen such that their corresponding dimensional parameters of $\nu, k_{0}$ and $k_{2}^{\prime}$ are equal to those used in the previous section, for the case of fast electrode kinetics, but with the radius of the electrode set to $1 \mu \mathrm{m}$ rather than $1 \mathrm{~mm}$; these dimensional parameters are, namely, $\nu=1 \mathrm{~V} / \mathrm{s}, k_{0}=10 \mathrm{~cm} / \mathrm{s}$ and $k_{2}^{\prime}=10^{1}-10^{11} \mathrm{M}^{-1} \mathrm{~s}^{-1}$. The diffusion coefficients and initial concentrations of A are considered to be the same in both cases as $D=10^{-5} \mathrm{~cm}^{2} \mathrm{~s}^{-1}$ and $c_{A}^{*}=1 m M$ respectively. This is done to best facilitate comparison of the results between the two diffusion regimes. The case of slow electrode kinetics is not explored as the following kinetics do not have an impact on an electrochemically 'irreversible' system other than diminishing the back-peak, as has been observed previously.

It is common in both theoretical ${ }^{5}$ and experimental studies ${ }^{31-35}$ to analyse the apparent transfer coefficient using a mass-transport-corrected Tafel plot ${ }^{15}$ for uniformly-accesible electrodes exhibiting steady state currents, such as micro-spheres, rather than a standard Tafel plot of $\ln |J|$ against $\theta$. As such we have used the same correction in our analysis of the effect of following kinetics on the apparent transfer coefficient for the micro-sphere regime. Plots of $\alpha_{\text {apparent }}=-\frac{d \ln (|J|)}{d \theta}$ against $\theta$ in the convergent $/$ divergent diffusion limit are presented in the Supplementary Information.

For a cathodic current, the mass-transport-corrected Tafel plot is $\ln \left|\frac{1}{J}-\frac{1}{J_{l i m}}\right|$ against $\theta$ and the value of $\alpha_{\text {apparent }}$ is extracted from the gradient:

$$
\alpha_{\text {apparent }}=\frac{d \ln \left|\frac{1}{J}-\frac{1}{J_{\text {lim }}}\right|}{d \theta}
$$

Figures 15(A), (C), and (E) display similar qualitative characteristics to their planar diffusion counterparts in Figures 10(B), 13(A), and 13(B). First, the increasing $K_{2}^{\prime}$ diminishes the back peak. Second, 

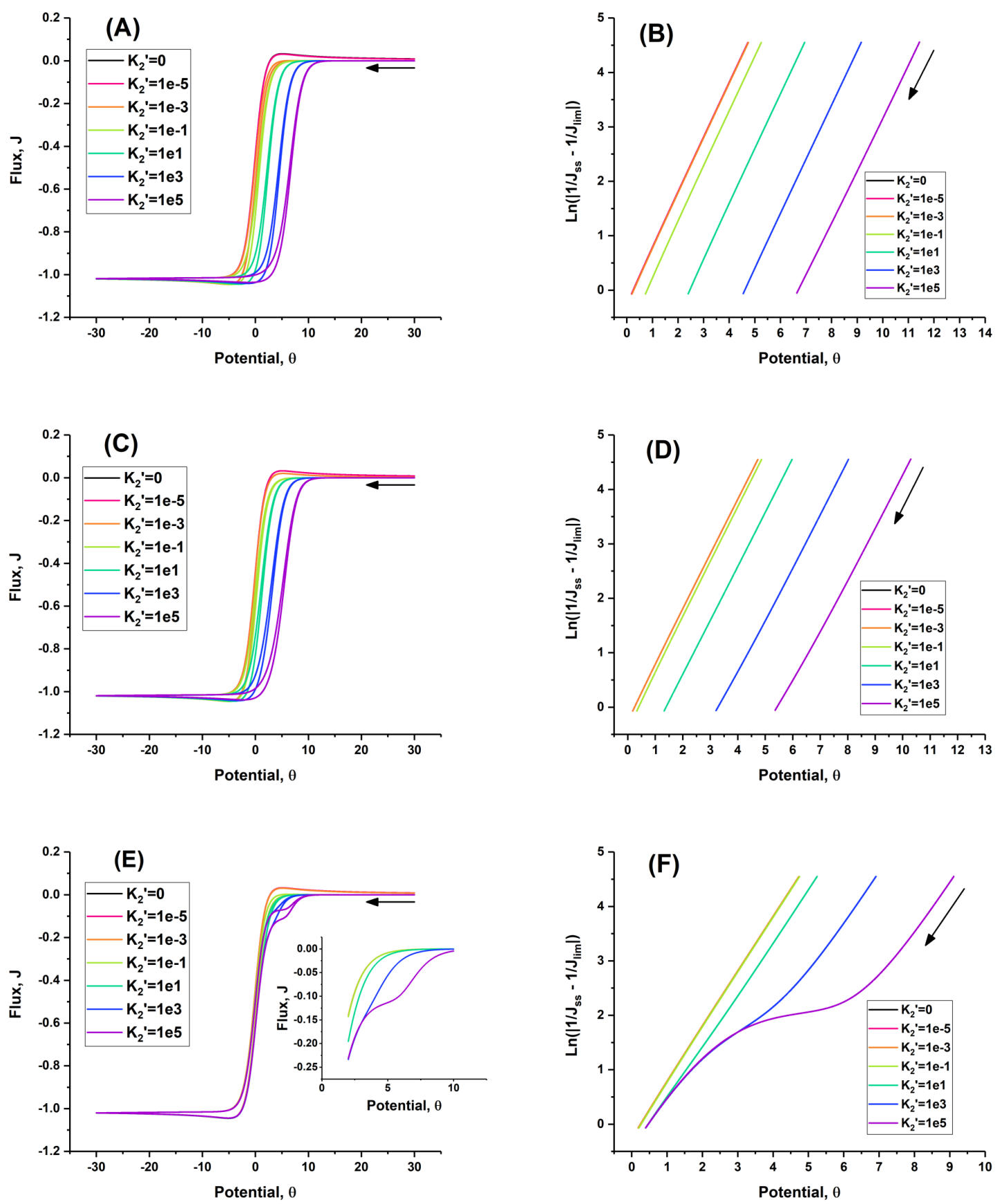

Figure 15: Figures (A), (C) and (E) show cyclic voltammograms for the 'titration' reaction under convergent/divergent diffusion with $C_{C}^{*}=10,1$, and 0.1 respectively. A relatively slow dimensionless scan rate of 0.038943 is chosen to correspond to a scan rate of $1 \mathrm{Vs}^{-1}$ on a $1 \mu \mathrm{m}$ radius electrode with diffusion coefficients of $D=10^{-5} \mathrm{~cm}^{2} \mathrm{~s}^{-1}$. To facilitate comparison with the results at the planar diffusion limit, $K_{0}$ is chosen to be $10^{2}$, which corresponds to the same $k_{0}$ on a $1 \mu \mathrm{m}$ electrode rather than a $1 \mathrm{~mm}$ electrode. Similarly, the $K_{2}^{\prime}$ range $\left(10^{-5}-10^{5}\right)$ is chosen such that it corresponds to the same dimensional range of $k_{2}^{\prime}\left(10^{1}-10^{11} M^{-1} s^{-1}\right)$ on a $1 \mu \mathrm{m}$ electrode as used on a $1 \mathrm{~mm}$ electrode for the investigation of the planar diffusion limit. Additionally, Figure (A) has an inset plot showing the forward scans at different $K_{2}^{\prime}$ between $\theta=2$ and $\theta=10$ to highlight the effect of the following kinetics at the beginning of the voltammetric curve. Figures (B), (D) and (F) show the corresponding mass-transport-corrected Tafel plots between 1 and $50 \%$ of the peak current for $C_{C}^{*}=10,1$, and 0.1 respectively. 
once $K_{2}^{\prime}$ has become relatively large, increasing $K_{2}^{\prime}$ shifts the reduction to more positive potentials, as the following kinetics perturb the electrochemical equilibrium earlier. Finally, for $C_{C}^{*}<1$, the depletion of $C_{C}$ causes a pre-peak for large $K_{2}^{\prime}$.

However there are some contrasting qualities. As expected for convergent/divergent diffusion, the current approaches a limiting value of $J_{l i m}=1$ at very negative potentials in the convergent/divergent diffusion regime; the following kinetics have no impact on the value of $J_{\text {lim }}$ if all species have equal diffusion coefficients. Furthermore, increasing $K_{2}^{\prime}$ has no apparent effect on the shape of the forward scan for $C_{C}^{*} \geq 1$ during the first part of the voltammetric curve; however the peak current does decrease slightly as $K_{2}^{\prime}$ increases at these $C_{C}^{*}$ values. For $C_{C}^{*} \geq 1$, diminishing the back peak removes the hysteresis between the forward and backward scans caused by residual planar contributions to the diffusion, causing the two to overlay at more positive potentials. For $C_{C}^{*}<1$, after the pre-peak has caused $\mathrm{C}$ to deplete, the forward voltammetric curve gradually returns to eventually overlay the curve of $K_{2}^{\prime}=0$.

Considering Figure 15(B), we see that increasing $K_{2}^{\prime}$ does not affect the shapes of the mass-transportcorrected Tafel plots, except that they are shifted to higher potentials as $K_{2}^{\prime}$ increases. This agrees with the observation that the forward scan of the corresponding cyclic voltammogram also does not change shape. In the mass-transport-corrected Tafel plot shown, $K_{2}^{\prime}=10^{-1}$ is the first to meaningfully deviate from $K_{2}^{\prime}=0$; thus it is around this value that the following kinetics are fast enough to impact the electrochemical system during the foward scan. The slopes maintain a gradient of 1 , irrespective of $K_{2}^{\prime}$, implying reversibility is maintained.

Figure 15(D) appears very similar to Figure 15(B), but the slopes are less shifted along the $\theta$-axis by the following kinetics. Again, $K_{2}^{\prime}=10^{-1}$ is the slowest to meaningfully deviate from $K_{2}^{\prime}=0$. Although the slopes appear linear, they do not maintain a gradient of 1 like those in Figure 15(B), they instead possess a weak curvature such that their gradients are initially equal to 1 but decrease at less positive potentials. The greater $K_{2}^{\prime}$ is, the greater the curvature. Thus there is a slight variation with $K_{2}^{\prime}$ in the shape of the forward scans of the cyclic voltammograms, which is negligible during the first half of the voltammetric curve. The curvature of the mass-transport-corrected Tafel plot is the result of $C_{C}$ depleting as the reaction progresses, thus weakening the driving force of the following kinetics to reduce A.

Figure 15(F) reveals greater subtlety once $C_{C}^{*}<1$ : similar to the other mass-transport-corrected Tafel plots, increasing $K_{2}^{\prime}$ shifts the initial slope to more positive potentials. However, for large $K_{2}^{\prime}$, the depletion of $\mathrm{C}$ has a marked effect. One clearly observes two regimes. The first regime comes at very positive potentials, before $\mathrm{C}$ experiences a significant depletion, where a gradient slightly below 
1 is observed, implying almost fully reversible reduction of A in this regime. As C depletes and the driving force of the chemical step to reduce A decreases, the curve goes through a transition and, due to the greatly decreased gradient in this region, arrives close to the slope of $K_{2}^{\prime}=0$. We then enter the second regime where the gradient increases to values just below 1 and the curve slowly approaches that of $K_{2}^{\prime}=0$. This regime corresponds with the contribution to the current from the unforced reduction of A outweighing the contribution from the reduction of A imposed by the chemical kinetics disturbing the electrochemical equilibrium, such that the mass transport corrected Tafel plot in this regime increasingly approaches a gradient of $\alpha_{\text {apparent }}=1$.

\section{Conclusions}

Tafel analysis is a routine and insightful component of voltammetric analysis. Whilst the interpretation in the limit of full electrochemical irreversibility is relatively simple, the simulations reported in this paper reveal marked complexity for faster electron transfer when coupled with non-linear chemical kinetics as evidenced for $\mathrm{EC}_{2}$ and 'titration' reactions, most notably the super-Nernstian behavior anticipated by the title of the paper and also the contrasting behavior of the titration reaction for macro and micro electrodes where simple mathematically analytical approches to data analysis lose their value. Rather we again ${ }^{36}$ conclude that the full simulation of experimental voltammograms is essential for even qualitative insight, as well as, of course, extraction of quantitative data.

\section{References}

[1] J. Tafel. Über die Polarisation bei kathodischer Wasserstoffentwicklung. Zeitshrift für Physikalische Chemie, 50(1):641-712, 1905.

[2] G. T. Burstein. A hundred years of Tafel's Equation: 1905-2005. Corrosion Science, 47(12):28582870,2005

[3] R. Guidelli, R. G. Compton, J. M. Feliu, E. Gileadi, J. Lipkowski, W. Schmickler, and S. Trasatti. Defining the transfer coefficient in electrochemistry: An assessment (IUPAC Technical Report). Pure and Applied Chemistry, 86(2):245-258, 2014.

[4] R. Guidelli, R. G. Compton, J. M. Feliu, E. Gileadi, J. Lipkowski, W. Schmickler, and S. Trasatti. Definition of the transfer coefficient in electrochemistry (IUPAC Recommendations 2014). Pure and Applied Chemistry, 86(2):259-262, 2014. 
[5] D. Li, C. Lin, C Batchelor-McAuley, L. Chen, and R. G. Compton. Tafel analysis in practice. Journal of Electroanalytical Chemistry, 826:117-124, 2018.

[6] M. C. Henstridge, E. Laborda, N. V. Rees, and R. G. Compton. Marcus-Hush-Chidsey theory of electron transfer applied to voltammetry: A review. Electrochimica Acta, 84:12-20, 2012.

[7] J. M. Savéant and D. Tessier. Non-Volmerian charge transfers associated with follow-up chemical reactions. A convolution potential sweep voltammetric study of benzaldehyde reduction in ethanol. The Journal of Physical Chemistry, 82(15):1723-1727, 1978.

[8] S. Fletcher and T. S. Varley. Beyond the Butler-Volmer equation. Curved Tafel slopes from steadystate current-voltage curves. Physical Chemistry Chemical Physics, 13(12):5359-5364, 2011.

[9] C. E. D. Chidsey. Free energy and temperature dependence of electron transfer at the metalelectrolyte interface. Science, 251(4996):919, 1991.

[10] H. O. Finklea and D. D. Hanshew. Electron-transfer kinetics in organized thiol monolayers with attached pentaammine(pyridine)ruthenium redox centers. Journal of the American Chemical Society, 114(9):3173-3181, 1992.

[11] L. Tender, M. T. Carter, and R. W. Murray. Cyclic Voltammetric Analysis of Ferrocene Alkanethiol Monolayer Electrode Kinetics Based on Marcus Theory. Analytical Chemistry, 66(19):3173-3181, 1994.

[12] R. A. Marcus. On the Theory of Electron-Transfer Reactions. VI. Unified Treatment for Homogeneous and Electrode Reactions. The Journal of Chemical Physics, 43(2):679-701, 1965.

[13] N. S. Hush. Adiabatic Rate Processes at Electrodes. I. Energy-Charge Relationships. The Journal of Chemical Physics, 28(5):962-972, 1958.

[14] E. E. L. Tanner, E. O. Barnes, C. B. Tickell, P. Goodrich, C. Hardacre, and R. G. Compton. Application of asymmetric Marcus-Hush theory to voltammetry in room-temperature ionic liquids. The Journal of Physical Chemistry C, 119(13):7360-7370, 2015.

[15] W. J. Albery. Electrode kinetics. Oxford chemistry series. Clarendon Press, 1975.

[16] R. P. Bacil, L. Chen, S. H. P. Serrano, and R. G. Compton. Dopamine oxidation at gold electrodes: mechanism and kinetics near neutral pH. Physical Chemistry Chemical Physics, 22(2):607-614, 2020. 
[17] I. Streeter, S. F. Jenkinson, G. W. J. Fleet, and R. G. Compton. Chemical instability promotes apparent electrochemical irreversibility: Studies on the electrode kinetics of the one electron reduction of the 2,6-diphenylpyrylium cation in acetonitrile solution. Journal of Electroanalytical Chemistry, 600(2):285-293, 2007.

[18] E. R. Lowe, C. E. Banks, and R. G. Compton. Indirect detection of substituted phenols and cannabis based on the electrochemical adaptation of the Gibbs reaction. Anal Bioanal Chem, 383(3):523-31, 2005.

[19] J. K. Novev and R. G. Compton. Natural convection effects in electrochemical systems. Current Opinion in Electrochemistry, 7:118-129, 2018.

[20] A. Einstein. On the motion of small particles suspended in liquids at rest required by the molecularkinetic theory of heat. Annalen der Physik, 17:549-560, 1905.

[21] I. B. Svir, A. I. Oleinick, and R. G. Compton. Solution of ring electrode problems in spherical coordinates: An application to near-steady-state linear sweep voltammetry. Russian Journal of Electrochemistry, 39(2):160-164, 2003.

[22] D. J. Gavaghan. An exponentially expanding mesh ideally suited to the fast and efficient simulation of diffusion processes at microdisc electrodes. 1. Derivation of the mesh. Journal of Electroanalytical Chemistry, 456(1-2):1-12, 1998.

[23] R. G. Compton and C. E. Banks. Understanding voltammetry. World Scientific, Third edition, 2018.

[24] E. Kätelhön and R. G. Compton. Testing and validating electroanalytical simulations. Analyst, 140(8):2592-2598, 2015.

[25] E. Kätelhön and R. G. Compton. Correction: Testing and validating electroanalytical simulations. Analyst, 140:3290-3290, 2015.

[26] E. Kätelhön and R. G. Compton. Correction: Testing and validating electroanalytical simulations. Analyst, 141(3):1154-1154, 2016.

[27] J. E. B. Randles. A cathode ray polarograph. part ii.- the current-voltage curves. Trans. Faraday Soc., 44:327-338, 1948.

[28] A Ševčik. Oscillographic polarography with periodical triangular voltage. Collection of Czechoslovak Chemical Communications, 13:349-377, 1948. 
[29] A. Savitzky and M. J. E. Golay. Smoothing and differentiation of data by simplified least squares procedures. Analytical Chemistry, 36(8):1627-1639, 1964.

[30] M. Jakubowska and W. W. Kubiak. Optimization of smoothing process - the method to improve calibration in voltammetry: Part I. simulated voltammograms. Talanta, 62(3):583 - 594, 2004.

[31] N. V. Rees, J. A. Alden, R. A. W. Dryfe, B. A. Coles, and R. G. Compton. Voltammetry under high mass transport conditions: The high speed channel electrode and heterogeneous kinetics. The Journal of Physical Chemistry, 99:14813-14818, 1995.

[32] M. E. G. Lyons, W. Breen, and J. Cassidy. Ascorbic acid oxidation at polypyrrole-coated electrodes. Journal of the Chemical Society, Faraday Transactions, 87(1):115-123, 1991.

[33] T. R. Ralph, M. L. Hitchman, J. P. Millington, and F. C. Walsh. The reduction of l-cystine in hydrochloric acid at mercury drop electrodes. Journal of Electroanalytical Chemistry, 587(1):31 41, 2006.

[34] N. C. L. Wood, C. A. Paddon, F. L. Bhatti, T. J. Donohoe, and R. G. Compton. Mediated electron transfer from lithium investigated voltammetrically in tetrahydrofuran: why are some mediators more effective reducing reagents than others? Journal of Physical Organic Chemistry, 20(10):732$742,2007$.

[35] L. Chen, E. E. L. Tanner, C. Lin, and R. G. Compton. Impact electrochemistry reveals that graphene nanoplatelets catalyse the oxidation of dopamine via adsorption. Chemical Science, 9:152-159, 2018.

[36] D. Li, C. Batchelor-McAuley, and R. G. Compton. Some thoughts about reporting the electrocatalytic performance of nanomaterials. Applied Materials Today, 18:100404, 2020. 